\title{
Spin-phonon coupling in single Mn-doped CdTe quantum dot
}

\author{
C. L. Cao, ${ }^{1,2}$ L. Besombes, ${ }^{2}$ and J. Fernández-Rossier ${ }^{1,3}$ \\ ${ }^{1}$ Departamento de Física Aplicada, Universidad de Alicante, San Vicente del Raspeig, E-03690 Spain \\ ${ }^{2}$ CEA-CNRS group "Nanophysique et semiconducteurs," Institut Néel, CNRS and Université Joseph Fourier, B.P. 166, \\ F-38042 Grenoble Cedex 9, France \\ ${ }^{3}$ International Iberian Nanotechnology Laboratory, Avenida Mestre José Veiga, P-4715-330 Braga, Portugal
}

(Received 15 June 2011; revised manuscript received 29 September 2011; published 10 November 2011)

\begin{abstract}
The spin dynamics of a single Mn atom in a laser driven CdTe quantum dot is addressed theoretically. Recent experimental results [Gall et al., Phys. Rev. Lett. 102, 127402 (2009); Goryca et al., Phys. Rev. Lett. 103, 087401 (2009); Gall et al., Phys. Rev. B 81, 245315 (2010)] show that it is possible to induce Mn spin polarization by means of circularly polarized optical pumping. Pumping is made possible by the faster Mn spin relaxation in the presence of the exciton. Here we discuss different Mn spin-relaxation mechanisms: first, Mn-phonon coupling, which is enhanced in the presence of the exciton; second, phonon induced hole spin relaxation combined with carrier-Mn spin-flip coupling and photon emission results in Mn spin relaxation. We model the Mn spin dynamics under the influence of a pumping laser that injects excitons into the dot, taking into account exciton-Mn exchange and phonon induced spin relaxation of both $\mathrm{Mn}$ and holes. Our simulations account for the optically induced $\mathrm{Mn}$ spin pumping.
\end{abstract}

DOI: 10.1103/PhysRevB.84.205305

PACS number(s): 76.60.Es, 81.07.Ta, 75.75.-c

\section{INTRODUCTION}

The tremendous progress in the miniaturization of electronic devices has reached the point that makes it crucial to address the effect of a single dopant in a device and motivates the study of a single dopant spin to store digital information. ${ }^{1}$ The manipulation of a single atom spin in a solid-state environment has been demonstrated using several approaches, like scanning tunneling microscope on magnetic adatoms, ${ }^{2,3}$ or optical probing of nitrogen vacancy (NV) centers in diamond ${ }^{4}$ and single magnetic atoms in semiconductor quantum dots, the topic of this paper. Single quantum dots doped with a single $\mathrm{Mn}$ atom can be probed by means of single exciton spectroscopy in photoluminescence (PL) experiments. This has been done both in II-VI (Refs. 5-15) and III-V (Refs. 16 and 17) materials. In the case of single Mn-doped CdTe dots, information about the quantum spin state of a single $\mathrm{Mn}$ atom is extracted from the single exciton quantum dot photoluminescence due to the one on one relation between photon energy and polarization and the electronic spin state of the Mn atom. ${ }^{18-26}$ This has made it possible to measure the spin-relaxation time of a single $\mathrm{Mn}$ atom in a quantum dot under optical excitation, using photon autocorrelation measurements, ${ }^{14}$ and to realize the optical initialization and readout of the spin of the Mn atom. ${ }^{5-7}$

The observation of $\mathrm{Mn}$ spin orientation under quasiresonant optical pumping ${ }^{5-7}$ can be accounted for if the Mn spinrelaxation time is shorter in the presence of a quantum dot exciton. ${ }^{7,27-29}$ In that situation, resonant excitation of an optical transition associated to a given $\mathrm{Mn}$ spin projection results in the depletion of the laser driven Mn spin state, via Mn spin relaxation in the presence of the exciton. Whereas theoretical understanding of the exchange couplings between electrons, holes, and Mn spin in quantum dots permits us to account for the observed PL spectra, ${ }^{8,10,11,18}$ a complete understanding of the spin dynamics under the combined action of laser pumping, incoherent spin relaxation, and coherent spin flips is still missing. In this paper we make progress along this direction on two counts. First, we discuss different Mn spin-relaxation mechanisms, taking fully into account the interplay between incoherent dynamics due to the coupling to a reservoir and the coherent spin flips associated to exciton-Mn exchange in the quantum dot. Our calculations show that the most efficient Mn spin-relaxation channel, in the presence of the exciton, arises from a combination of phonon induced hole spin relaxation, which turns the bright exciton into a dark, followed by recombination to the ground enabled by dark-bright mixing due to Mn-carrier spin-flip exchange. Thus we provide a quantitative basis to a recently proposed scenario. ${ }^{29}$ Second, we model the Mn spin dynamics with a rate equation for the Mn spin and the Mn plus exciton spin states that includes the spin-relaxation rates between the few-body states calculated from microscopic theory.

Our theory permits us to model the experimental observations and, importantly, it identifies the light-hole heavy-hole mixing as a crucial parameter that determines not only the PL line shape ${ }^{10,11,18}$ but also the amplitude of several spinrelaxation mechanisms at play in this system.

The rest of this paper is organized as follows. In Sec. II we review the Hamiltonian for a single Mn spin interacting with a single exciton in a quantum dot. The anisotropic Mnhole coupling is derived from a simplified ${ }^{18,30}$ single-particle description of the lowest energy quantum dot hole states, which affords analytical expressions for the critical parameter in the theory, the light-hole-heavy-hole (LH-HH) mixing. ${ }^{30}$ The dependence of the properties of the Mn-exciton states on the LH-HH mixing are discussed. In Sec. III we discuss the Mn spin relaxation due to Mn-phonon coupling, both with and without an exciton in the quantum dot. Whereas this mechanism is probably dominant for the Mn spin relaxation in the optical ground state, it is not sufficient to account for the rapid $\mathrm{Mn}$ spin relaxation in the presence of the exciton. This leads us to consider other spin-relaxation mechanisms. In Sec. IV we describe the spin relaxation of holes due to their coupling to acoustic phonons, using a Bir-Pikus Hamiltonian. Using the simplified description of hole states, we obtain 
analytical results for the hole spin lifetime in a nonmagnetic dot, which are in agreement with previous work using a more sophisticated description of single hole states. ${ }^{31}$ We then compute the lifetime of the exciton-Mn states due to hole spin relaxation. In Sec. VI we present our simulations of the optical pumping process, using rate equations for the exciton-Mn quantum states, including the laser pumping, the spontaneous photon emission, and the $\mathrm{Mn}$ and hole spin relaxation due to phonons. Our simulations account for the optical initialization and readout observed experimentally.

\section{EXCITON-Mn HAMILTONIAN}

In this section we describe a minimal Hamiltonian model that can accounts for the PL spectra of single Mn-doped CdTe quantum dots. For that matter we need to consider both the Mn spin in the unexcited crystal and the Mn spin interacting with a quantum dot exciton. The peaks in the PL spectra are associated to the energy differences between the states of the dot with and without the exciton.

\section{A. Mn spin Hamiltonian}

$\mathrm{Mn}$ is a substitutional impurity in the Cd site of CdTe. Thus it has an oxidation state of $2+$, so that the $5 d$ electrons have spin $S=\frac{5}{2}$, resulting in a sextuplet ${ }^{32}$ whose degeneracy is lifted by the interplay of spin orbit and the crystal field. In an unstrained CdTe, the crystal field has cubic symmetry, which should result in a magnetic anisotropy Hamiltonian without quadratic terms. Electron paramagnetic resonance (EPR) in CdTe strained epilayers ${ }^{33}$ show that Mn has an uniaxial term in the spin Hamiltonian. In a quantum dot there could be some in-plane anisotropy as well, which lead us to consider the following Hamiltonian:

$$
\mathcal{H}_{0}=D M_{z}^{2}+E\left(M_{x}^{2}-M_{y}^{2}\right)+g \mu_{B} \vec{B} \cdot \vec{M},
$$

where $M_{a}$ are the $S=\frac{5}{2}$ spin operators of the electronic spin of the Mn. The eigenstates of this Hamiltonian are denoted by $\phi_{m}$,

$$
\mathcal{H}_{0}\left|\phi_{m}\right\rangle=E_{m}\left|\phi_{m}\right\rangle=E_{m} \sum_{M_{z}} \phi_{m}\left(M_{z}\right)\left|M_{z}\right\rangle,
$$

where $\left|M_{z}\right\rangle$ are the eigenstates of $M_{z}$. In this paper we neglect the hyperfine coupling to the $I=\frac{5}{2}$ nuclear spin, which could affect the decay of the electronic-spin coherence. ${ }^{5}$ The magnetic anisotropy parameters $E$ and $D$ cannot be inferred from PL experiments, which are only sensitive to the Mn-exciton coupling. EPR experiments ${ }^{33}$ in strained layer could be fit with $D=12 \mu \mathrm{eV}, E=0$, and $g=2.0$. Thus the ground state should have $M_{z}= \pm \frac{1}{2}$, split from the first excited state by $2 D$. At $4 \mathrm{~K}$ and zero magnetic field, we expect all the six spin levels to be almost equally populated. We refer to these six states as the ground-state manifold, in contrast to the excited-state manifold, which we describe with 24 states corresponding to four possible quantum dot exciton states and the six Mn spin states.

\section{B. Single-particle states of the quantum dot}

We describe the confined states of the quantum dot with a simple effective-mass model. In the case of the conduction-band electrons, we neglect spin-orbit coupling and we only consider the lowest energy orbital, with wave function $\psi_{0}(\vec{r})$, which can accommodate one electron with two spin orientations.

In the case of holes, spin-orbit coupling lifts the sixfold degeneracy of the top of the valence band into a $J=\frac{3}{2}$ quartet and a $J=\frac{1}{2}$ doublet which, in CdTe, is more than $0.8 \mathrm{eV}$ below in energy. Confinement and strain lift the fourfold degeneracy of the $J=\frac{3}{2}$ hole states, giving rise to a mostly $J_{z}= \pm \frac{3}{2}$ heavy-hole doublet and an almost $J_{z}= \pm \frac{1}{2}$ light-hole doublet. Importantly, it is crucial to include LH-HH mixing to describe the experimental observation.

\section{Effect of confinement}

The top of the valence-band states are described in the $k p$ approximation with the so-called Kohn-Luttinger (KL) Hamiltonian. ${ }^{34-36}$ For that matter, the crystal Hamiltonian is represented in the basis of the four topmost $J=\frac{3}{2}$ valence states of the $\Gamma$ point. We label them by $J=\frac{3}{2}, J_{z}$. The resulting kp Hamiltonian can be written as $\mathcal{H}_{\text {holes }}=\mathcal{H}_{\mathrm{KL}}$,

$$
\mathcal{H}_{\mathrm{KL}}=\sum_{i, j=x, y, z} V_{i j}^{\mathrm{KL}}(\vec{k}) J_{i} J_{j}+\kappa \mu_{B} J_{z} B,
$$

where $\vec{k}$ is the Bloch wave vector, $J_{i}$ are the spin $\frac{3}{2}$ matrices, and $V_{i j}^{\mathrm{KL}}$ are coefficients given in Appendix A, which are quadratic in the components of $\vec{k}$. The last term accounts for the Zeeman coupling to an external field along the growth direction.

The $k p$ Hamiltonian for states in the presence of a quantum dot confinement potential that breaks translational invariance reads, for zero applied field,

$$
\mathcal{H}_{\mathrm{kp}}=-\hbar^{2} \sum_{i, j=x, y, z} V_{i j}^{\mathrm{KL}}\left(\partial_{a} \partial_{b}\right) J_{i} J_{j}+V(\vec{r}) \delta_{j_{z}, j_{z}^{\prime}},
$$

where the matrix elements of $V_{i j}^{\mathrm{KL}}\left(\partial_{a} \partial_{b}\right)$ are a second-order differential operators and $a, b=x, y, z$. In general, the numerical solution of Eq. (4) can be very complicated. Following previous work, ${ }^{18,30}$ we make two approximations that permit us to obtain analytical solutions. First, we model the quantum dot with a hard-wall box-shape potential. The dimensions of the box are $L_{x}, L_{y}$, and $L_{z}$. This permits us to write the wave function as a linear combination of $\left|J=\frac{3}{2}, J_{z}\right\rangle$ states multiplied by the confined waves

$$
\psi_{\vec{n}}(\vec{r})=\sqrt{\frac{8}{V}} \sin \left(\frac{n_{x} \pi x}{L_{x}}\right) \sin \left(\frac{n_{y} \pi y}{L_{y}}\right) \sin \left(\frac{n_{z} \pi z}{L_{z}}\right) .
$$

Our second approximation is to restrict the basis set to the fundamental mode only, $n_{x}=n_{y}=n_{z}=1$. As a result, the quantum dot Hamiltonian reads

$$
\mathcal{H}_{\mathrm{kp}}=-\hbar^{2} \sum_{i, j=x, y, z} V_{i j}^{\mathrm{KL}}\left(\left\langle\partial_{a} \partial_{b}\right\rangle\right) J_{i} J_{j},
$$

where

$$
\left\langle\partial_{a} \partial_{b}\right\rangle=\int \psi_{1,1,1}(\vec{r}) \partial_{a} \partial_{b} \psi_{1,1,1}(\vec{r})=\delta_{a b}\left(\frac{2 \pi}{L_{a}}\right)^{2} .
$$


Thus, within this approximation, the quantum dot hole states are described by a $4 \times 4$ Kohn Luttinger Hamiltonian where the terms linear in $k_{i}$ vanish and the $k_{i}^{2}$ terms are replaced by $\left(\frac{2 \pi}{L_{i}}\right)^{2}$. The resulting matrix $\mathcal{H}_{\text {conf }}$ has two decoupled sectors denoted by $+\left(J_{z}=+\frac{3}{2}, J_{z}=-\frac{1}{2}\right)$ and $-\left(J_{z}=-\frac{3}{2}, J_{z}=+\frac{1}{2}\right)$. In the $\left(+\frac{3}{2},-\frac{1}{2},+\frac{1}{2},-\frac{3}{2}\right)$ basis we have

$$
\mathcal{H}_{\text {conf }}=\left(\begin{array}{cc}
\mathcal{H}_{+} & 0 \\
0 & \mathcal{H}_{-}
\end{array}\right)
$$

with

$$
\mathcal{H}_{+}=\left(\begin{array}{cc}
\bar{P}+\frac{\bar{Q}}{\bar{R}}-\frac{3 b}{2} & \bar{P}-\overline{\bar{Q}}+\frac{b}{2}
\end{array}\right)
$$

and

$$
\mathcal{H}_{-}=\left(\begin{array}{cc}
\bar{P}-\frac{\bar{Q}-\frac{b}{2}}{P}+\bar{Q} & \bar{Q}+\frac{3 b}{2}
\end{array}\right),
$$

where $b \equiv \kappa \nu_{B} B$ and $\bar{P}, \bar{Q}$, and $\bar{R}$ are given in Appendix A. In order to find the corresponding energies and wave functions it is convenient to write these matrices as $\mathcal{H}_{ \pm}=a_{ \pm}+\vec{h}_{ \pm} \cdot \vec{\sigma}$ where $\vec{\sigma}$ are the Pauli matrices acting on the pseudospin $\frac{1}{2}$ space of the + and - spaces, $a_{ \pm}=P \mp b / 2$ and

$$
\vec{h}_{ \pm}=(\bar{R}, 0, \bar{Q} \mp b)=\left|\vec{h}_{ \pm}\right|\left(\sin \theta_{ \pm}, 0, \cos \theta_{ \pm}\right) .
$$

We keep only the two ground states (heavy-hole like), denoted by $|\Uparrow\rangle$ and $|\Downarrow\rangle$, which are several meV away from the lighthole-like states. The ground-state doublet for the quantum dot holes states so obtained, neglecting strain, can be written as

$$
\begin{aligned}
& |\Uparrow\rangle=\cos \frac{\theta_{+}}{2}\left|+\frac{3}{2}\right\rangle+\sin \frac{\theta_{+}}{2}\left|-\frac{1}{2}\right\rangle, \\
& |\Downarrow\rangle=\cos \frac{\theta_{-}}{2}\left|+\frac{-3}{2}\right\rangle+\sin \frac{\theta_{-}}{2}\left|+\frac{1}{2}\right\rangle .
\end{aligned}
$$

Thus the LH-HH mixing parameters $\theta_{ \pm}$depend on the dot dimension $L_{i}$ on the Kohn Luttinger parameters $\gamma_{i}$ and on the applied magnetic field $B$.

Whereas in most instances the elimination of the two higher energy eigenstates of the hole Hamiltonian is a good approximation, there is a caveat to keep in mind. Let us consider the single-hole states in Eq. (12) at finite field and expand them in terms of the zero-field ( $\left.b=0, \theta_{+}=\theta_{-}\right)$basis. It is apparent that the effect of the magnetic field is to mix the low- and high-energy sectors (as defined at zero field). In addition, this mixing is different for the two sectors denoted by \pm . As we show below, hole-spin relaxation is possible at finite magnetic field exactly because of this channel dependent mixing. At zero magnetic field exchange coupling of the hole to $\mathrm{Mn}$ and electrons has the same effect, but, by keeping only the low-energy states (12) in the zero-field basis, the effect is not captured.

\section{Effect of homogeneous strain}

We now consider the effect of the strain that arises from the lattice mismatch between the CdTe quantum dot and the ZnTe substrate on the $J=\frac{3}{2}$ states of the valence band. It has a similar effect as confinement, resulting in a splitting of the $J=\frac{3}{2}$ manifold and a mixing of the LH and HH states. The Hamiltonian that describes the effect of strain, as described by the strain tensor $\epsilon_{i j}$, on the top of the valence-band states in zinc-blende semiconductors was proposed by Bir and Pikus. We can write the Bir and Pikus (BP) Hamiltonian as ${ }^{37}$

$$
\begin{aligned}
\mathcal{H}_{\mathrm{BP}}= & \left(a-\frac{9 b}{4}\right)\left(e_{x x}+e_{y y}+e_{z z}\right)+b \sum_{i=x, y, z} J_{i}^{2} e_{i i} \\
& +\frac{d}{\sqrt{3}}\left[\left(J_{x} J_{y}+J_{y} J_{x}\right) e_{x y}+\text { c.p. }\right],
\end{aligned}
$$

where c.p. stands for cyclic permutation, and $a=-0.91 \mathrm{eV}$, $b=-1.2 \mathrm{eV}, d=-5.4 \mathrm{eV}$ for CdTe. ${ }^{37}$

For CdTe quantum dots grown in $\mathrm{ZnTe}$, we mainly consider the effects of strain anisotropy in the growth plane ${ }^{13}$ and describe the strain by the average values of $e_{x y}$ and $e_{x x}-e_{y y}$. In this approximation the BP Hamiltonian is reduced to a block diagonal matrix in the $\left(+\frac{3}{2},-\frac{1}{2},+\frac{1}{2},-\frac{3}{2}\right)$ basis:

$$
\mathcal{H}_{B P}=\left(\begin{array}{cc}
\mathcal{H}_{B P+} & 0 \\
0 & \mathcal{H}_{B P-}
\end{array}\right)
$$

where

$$
\begin{aligned}
& \mathcal{H}_{B P+}=\left(\begin{array}{lc}
0 & \rho_{s} e^{-2 i \varphi_{s}} \\
\rho_{s} e^{2 i \varphi_{s}} & \Delta_{l h}
\end{array}\right), \\
& \mathcal{H}_{B P-}=\left(\begin{array}{cc}
\Delta_{l h} & \rho_{s} e^{-2 i \varphi_{s}} \\
\rho_{s} e^{2 i \varphi_{s}} & 0
\end{array}\right),
\end{aligned}
$$

where $\Delta_{l h}=b\left(e_{x x}+e_{y y}\right)$ is the strain induced HH-LH splitting, $\rho_{s}$ is the strain induced amplitude of the HH-LH mixing, and $\phi_{s}$ is the angle between the strain induced anisotropy axis in the quantum dot plane and the $x(100)$ axis:

$$
\rho_{s} e^{-2 i \varphi_{s}}=\frac{\sqrt{3}}{2} b\left(e_{x x}-e_{y y}\right)-i d e_{x y} .
$$

Importantly, the effect of confinement and the effect of strain have a very similar mathematical structure. They both split and mix the $\mathrm{LH}$ and $\mathrm{HH}$ levels. The main difference lies in the mixing term, which is real for the confinement Hamiltonian controlled by the shape of the quantum dot and complex for the BP Hamiltonian depending on the strain distribution in the quantum dot plane.

\section{Combined effect of confinement and strain}

We finally consider the combined action of confinement and strain described by $\mathcal{H}_{\text {holes }}=\mathcal{H}_{\text {conf }}+\mathcal{H}_{B P}$. Summing the Hamiltonians of Eqs. (8) and (14) we obtain two decoupled matrices for the + and - subspaces. They can be written as

$$
\mathcal{H}_{\text {tot }, \pm}=A_{ \pm}+\vec{H}_{ \pm} \cdot \vec{\sigma}
$$

where $A_{ \pm}=\bar{P} \mp \frac{b}{2}+\frac{\Delta_{l h}}{2}$ and

$$
\vec{H}_{ \pm}=\left(\bar{R}+\rho_{s} \cos \left(2 \varphi_{s}\right), \pm \rho_{s} \sin \left(2 \varphi_{s}\right), \bar{Q} \mp b-\frac{\Delta_{l h}}{2}\right) \text {. }
$$

It is convenient to express the ground-state doublet associated to $\mathcal{H}_{\text {holes }}$ in terms of the spherical coordinates of the vectors 


$$
\begin{aligned}
\vec{H}_{ \pm},\left|\vec{H}_{ \pm}\right|, \theta_{ \pm}, \text {and } \phi_{ \pm}: & \\
|\uparrow\rangle & =\cos \frac{\theta_{+}}{2}\left|\frac{+3}{2}\right\rangle-\sin \frac{\theta_{+}}{2} e^{i \phi_{+}}\left|\frac{-1}{2}\right\rangle, \\
|\Downarrow\rangle & =\cos \frac{\theta_{-}}{2}\left|\frac{-3}{2}\right\rangle-\sin \frac{\theta_{-}}{2} e^{i \phi_{-}}\left|\frac{+1}{2}\right\rangle,
\end{aligned}
$$

where

$$
e^{i \phi_{ \pm}}=\frac{\bar{R}+\rho_{s} e^{ \pm 2 i \varphi_{s}}}{\left|\bar{R}+\rho_{s} e^{ \pm 2 i \varphi_{s}}\right|} .
$$

Expectedly, this expression is formally very similar to that of Eq. (12).

Formally, we express Eq. (20) as

$$
\left|\sigma_{h}\right\rangle=\sum_{j_{z}} C_{h}\left(j_{z}\right)\left|j_{z}\right\rangle
$$

Both in Eqs. (12) and (20) the $\left(+\frac{3}{2},-\frac{1}{2}\right)$ sector is decoupled from the $\left(-\frac{3}{2},+\frac{1}{2}\right)$. Whereas this is not true in general, it is sufficient to account for the correct symmetry of a variety of exchange couplings between the hole and both the Mn and the electrons.

\section{Effective Mn-carrier exchange Hamiltonian}

\section{Hole-Mn Hamiltonian}

We now consider the exchange coupling of hole spin $(\vec{J})$ and the Mn spin $(\vec{M})$. The leading term in the exchange interaction is the Heisenberg operator, ${ }^{32,38}$

$$
\mathcal{V}_{\text {exch }}=\frac{1}{3} \beta \delta\left(\vec{r}_{h}-\vec{r}_{M}\right) \vec{J} \cdot \vec{M},
$$

where $\beta$ is the hole-Mn exchange coupling constant. For Mn in CdTe we have $\beta N_{0}=0.88 \mathrm{eV}$, where $N_{0}$ is the volume of the CdTe unit cell. ${ }^{32}$ The exchange interaction is taken as short ranged, the Mn atom is located at $\vec{r}_{\mathrm{Mn}}$, and $\vec{J}$ are the spin $\frac{3}{2}$ angular momentum matrices. We represent the operator (23) in the product basis $|M\rangle \times \sigma_{h}$. Thus, the exchange operator in the product basis reads:

$$
\begin{aligned}
& \left\langle M\left|\left\langle\sigma_{h}\left|\mathcal{V}_{\text {exch }}\right| M^{\prime}\right\rangle\right| \sigma_{h}^{\prime}\right\rangle \\
& \quad=\beta\left|\psi_{0}\left(\vec{r}_{M n}\right)\right|^{2} \sum_{a}\left\langle M\left|M_{a}\right| M^{\prime}\right\rangle \mid\left\langle\sigma_{h}\left|J_{a}\right| \sigma_{h}^{\prime}\right\rangle,
\end{aligned}
$$

where $\psi_{0}(\vec{r})$ is the envelope part of the heavy-hole wave function, Eq. (5), and $j_{h} \equiv \frac{1}{3} \beta\left|\psi_{0}\left(\vec{r}_{M n}\right)\right|^{2}$ is the hole-Mn coupling constant, which depends both on a material dependent constant $\beta$ and on a quantum dot dependent property, the probability of finding the hole at the Mn location.

After a straightforward calculation we obtain the effective Mn-hole coupling spin model working in the space $\left(M, \sigma_{h}\right)$ of dimension 12 as a function of the hole wave function parameter $\theta$ :

$$
\mathcal{V}_{h-M n}=j_{h x} M_{x} \sigma_{x}+j_{h y} M_{y} \sigma_{y}+j_{h z} M_{z} \sigma_{z},
$$

where the $j_{h i}$ are dimensionless coefficients given, for $B=0$ and $\theta=\theta_{+}=\theta_{-}$, by

$$
j_{h x}=\frac{j_{h}}{2}(\sqrt{3} \sin \theta+1-\cos \theta),
$$

$$
\begin{gathered}
j_{h y}=\frac{j_{h}}{2}(\sqrt{3} \sin \theta-1+\cos \theta), \\
j_{h z}=\frac{j_{h}}{2}(1+2 \cos \theta) .
\end{gathered}
$$

Notice that for $\theta=0$ there is no LH-HH mixing and we have $j_{h x}=j_{h y}=0$ and $j_{h z}=\frac{3}{2} j_{h}$. In this extreme case the Mn-hole coupling is Ising-like and $M_{z}$ and $\sigma_{z}$ are conserved. This limit is a good starting point to model hole-Mn coupling in CdTe quantum dots ${ }^{18,39}$

\section{Electron-Mn Hamiltonian}

In analogy to the hole-Mn bare coupling, the electron-Mn coupling reads

$$
\mathcal{V}_{\mathrm{e}-\mathrm{Mn}}=\alpha \delta\left(\vec{r}_{e}-\vec{r}_{M}\right) \vec{S} \cdot \vec{M},
$$

where $\vec{S}$ is the spin of the electron. Since the spin-orbit coupling has a very small effect on the $s$-like conduction band, the effective exchange for the quantum dot electron and the $\mathrm{Mn}$ is also a Heisenberg term given by

$$
\mathcal{V}_{\mathrm{e}-\mathrm{Mn}}=j_{e} \vec{S} \cdot \vec{M}=j_{e}\left(S_{x} M_{z}+S_{y} M_{y}+S_{z} M_{z}\right),
$$

where $j_{e} \equiv \alpha\left|\psi_{0}\left(\vec{r}_{M n}\right)\right|^{2}$ is the electron-Mn coupling constant, which depends both on a material dependent constant $\alpha$ and on a quantum dot dependent property, the probability of finding the electron at the Mn location. In the hard-wall model the orbital wave function for the confined electron and hole is the same, so that the ratio should be $j_{h} / j_{e} \simeq \frac{\beta}{3 \alpha}$ for CdTe. However, in experiments the ratio is bigger than that. ${ }^{8}$ In the following we take $j_{e}$ and $j_{h}$ as adjustable parameters.

\section{Exciton-Mn wave functions and energy levels}

\section{Hamiltonian}

The effective Hamiltonian for the exciton in a single Mn-doped CdTe quantum dot is the sum of the singleion magnetic anisotropy Hamiltonian, the Mn-electron and Mn-hole exchange coupling, and the electron-hole exchange coupling

$$
\mathcal{H}=\mathcal{H}_{S}+\mathcal{V}_{e-M n}+\mathcal{V}_{h-M n}+\mathcal{V}_{e-h},
$$

where

$$
\mathcal{V}_{e-h}=j_{e h} S_{z} \sigma_{h}
$$

is the electron-hole exchange coupling, neglecting transverse components. Electron-hole exchange is ferromagnetic $\left(j_{e h}<\right.$ 0 ) and splits the four exciton levels into two doublets, the low-energy dark doublet $(\Uparrow \uparrow, \Downarrow \downarrow)$, denoted by $X= \pm 2$, and the high-energy bright doublet $(\Uparrow \downarrow, \downarrow \uparrow)(X= \pm 1)$.

Since we consider two electron states $\left(S_{z}=\uparrow, \downarrow\right)$, two hole states $\left(\sigma_{h}=\Uparrow, \Downarrow\right)$, and six Mn states $M_{z}= \pm \frac{5}{2}, \pm \frac{3}{2}, \pm \frac{1}{2}$, the Hilbert space for the Mn-exciton system has dimension 24. Whereas we do obtain the exact eigenstates of Hamiltonian (31) by numerical diagonalization, it is convenient for the discussion to relate them to eigenstates of the Ising, or spin conserving, part of the Hamiltonian:

$$
\mathcal{H}=\mathcal{H}_{\text {Ising }}+\mathcal{H}_{\text {flip }}
$$


where

$$
\mathcal{H}_{\text {Ising }}=D M_{z}^{2}+j_{e h} S_{z} \sigma_{h}+j_{e} S_{z} M_{z}+j_{h} M_{z} \sigma_{h}
$$

and

$$
\begin{aligned}
\mathcal{H}_{\text {flip }}= & E\left(M_{x}^{2}-M_{y}^{2}\right)+j_{e}\left(S_{x} M_{x}+S_{y} M_{y}\right) \\
& +\left(j_{h x} \sigma_{x} M_{x}+j_{h y} \sigma_{y} M_{y}\right) .
\end{aligned}
$$

If we expand $j_{h x}$ and $j_{h y}$ in the series of LH-HH mixing parameter $\theta$, they are the same in the first order of $\theta$. For simplicity, we take

$$
j_{h \perp} \equiv j_{h x}=j_{h y}=j_{h} \frac{\theta}{2 \sqrt{3}}
$$

in the following calculation. In the case of a LH-HH mixing induced by the anisotropy of the confinement described by a hard-wall box-shape potential, we get from the Kohn-Luttinger Hamiltonian

$$
\theta=\frac{\pi^{2} \sqrt{3} \gamma_{2}\left|\frac{1}{L_{x}^{2}}-\frac{1}{L_{y}^{2}}\right|}{\sqrt{3 \pi^{4} \gamma_{2}^{2}\left(\frac{1}{L_{x}^{2}}-\frac{1}{L_{y}^{2}}\right)^{2}+\gamma_{1}^{2}\left(\frac{-2}{L_{z}^{2}}+\frac{1}{L_{x}^{2}}+\frac{1}{L_{y}^{2}}\right)^{2}}} .
$$
basis

The eigenstates of $\mathcal{H}_{\text {Ising }}$ are trivially given by the product

$$
|P\rangle \equiv\left|M_{z}\right\rangle\left|S_{z}\right\rangle\left|\sigma_{h}\right\rangle
$$

with eigenenergies

$$
E_{P}=D M_{z}^{2}+j_{e h} S_{z} \sigma_{h}+j_{e} S_{z} M_{z}+j_{h} M_{z} \sigma_{h} .
$$

Since the magnetic anisotropy term $D M_{z}^{2}$ is present both in the ground-state and exciton-state manifolds, it does not affect the PL spectra of the bright excitons. Within this picture, for each of the six possible values of $M_{z}$, there are four exciton states. We use a shorthand notation to refer to the Ising states $P_{X}\left(M_{z}\right)$ where $X= \pm 1, \pm 2$ labels the spin of the exciton, $X=S_{z}+\sigma_{z}$. An energy diagram for the exciton levels, within the Ising approximation, is shown in Fig. 1.

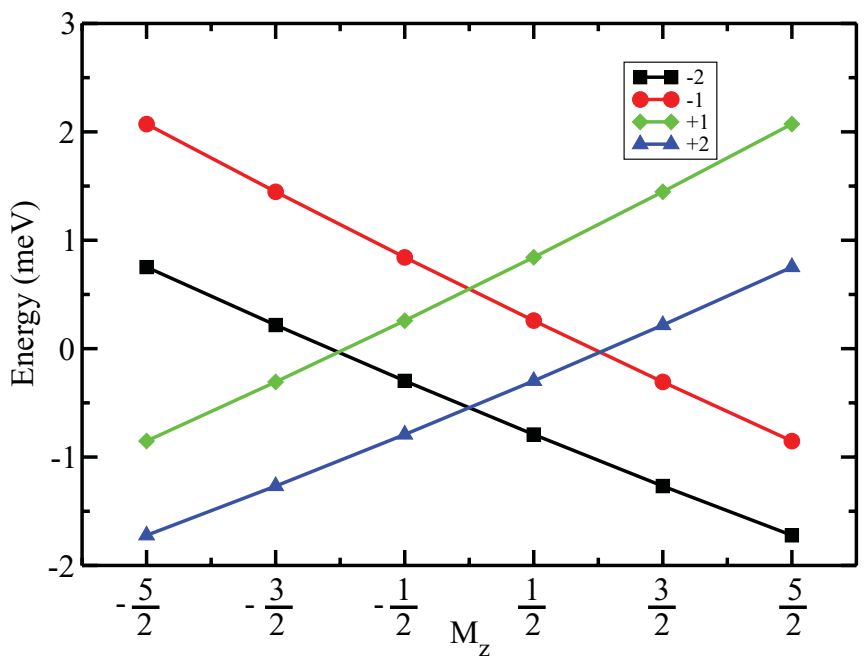

FIG. 1. (Color online) Scheme of the energy levels of the quantum dot exciton interacting with one $\mathrm{Mn}$ when spin-flip terms are neglected.
The PL spectra of a single Mn-doped quantum dot predicted by the model of Ising excitons, i.e., neglecting the spin-flip transitions, features six peaks corresponding to transitions conserving $M_{z}$. For the recombination of $\sigma^{+}$excitons $\left(S_{z}=\right.$ $\left.-\frac{1}{2}, \sigma_{h}=\Uparrow\right)$ the high-energy peak corresponds to $M_{z}=+\frac{5}{2}$ and the low-energy peak to $M_{z}=-\frac{5}{2}$ on account of the antiferromagnetic coupling between the hole and the Mn. In the case of $\sigma^{-}$excitons the roles are reversed, but the PL spectrum is identical at zero magnetic field.

\section{Wave functions}

When spin-flip terms are restored in the Hamiltonian, the $P$ states are no longer eigenstates, but they form a very convenient basis to expand the actual eigenstates of $\mathcal{H}$, denoted by $\left|\Psi_{n}\right\rangle$ :

$$
\left|\Psi_{n}\right\rangle=\sum_{P} \Psi_{n}(P)|P\rangle=\sum_{X, M_{z}} \Psi_{n}\left(X, M_{z}\right)\left|X, M_{z}\right\rangle .
$$

In most cases, there is a strong overlap between $\Psi_{n}$ and a single state $|P\rangle$. This is expected for several reasons. First, the single-ion in-plane anisotropy is probably much smaller than the uniaxial anisotropy, $D \gg E$. Second, the electron-hole exchange, which is the exchange energy in the system, splits the dark and bright levels. Thus both electron and hole spin flip due to the exchange with the Mn spin is inhibited because they involve coupling between energy split bright and dark excitons. In addition, the electron-Mn exchange is smaller than the hole-Mn exchange, whose spin-flip part is proportional to the LH-HH mixing and approximately ten times smaller than the Ising part. In order to quantify the degree of spin mixing of an exact exciton state $\Psi_{n}$, we define the inverse participation ratio

$$
\operatorname{IPR}_{n} \equiv \sum_{P}\left|\Psi_{n}(P)\right|^{4} .
$$

This quantity gives a measure of the delocalization of the state $\Psi_{n}$ on the space of product states of Eq. (38). In the absence of mixing of different $P$ states, we have $\mathrm{IPR}_{n}=1$. In the case of a state equally delocalized in the 24 states of the $P$ space, we would have $\Psi_{n}(P)=\frac{1}{\sqrt{24}}$ and $\operatorname{IPR}_{n}=\frac{1}{24}$.

In Fig. 2 we plot the evolution of both the energy (left panel) and the Inverse Participatio Ratio (IPR) (right panel) as a function of $J_{h \perp}$, the LH-HH mixing parameter, of four states denoted by their dominant component at $J_{h \perp}=0$. For our choice of exchange constants, two of them, $\left|+1,-\frac{3}{2}\right\rangle$ and $\left|-2,-\frac{1}{2}\right\rangle$, are almost degenerate at $J_{h \perp}=0$, which means that the hole-Mn exchange compensates the dark-bright splitting, and couple these states via a hole-Mn spin flip. As a result, their energy levels split linearly as a function of $J_{h \perp}$ and the wave functions have a large weight on the two product states for finite $J_{h \perp}$. In contrast, the other two levels shown in Fig. $2,\left|+1,-\frac{1}{2}\right\rangle$ and $\left|-2,-\frac{3}{2}\right\rangle$, are not coupled via hole-Mn spin flip. As a result, their energies shift as a function of $J_{h \perp}$ due to coupling to other states, and their IPR undergoes a minor change, reflecting moderate mixing.

\section{Exchange induced dark-bright mixing}

The most conspicuous experimentally observable consequence of the exchange induced mixing is the transfer of optical weight from the bright to the dark exciton, which 

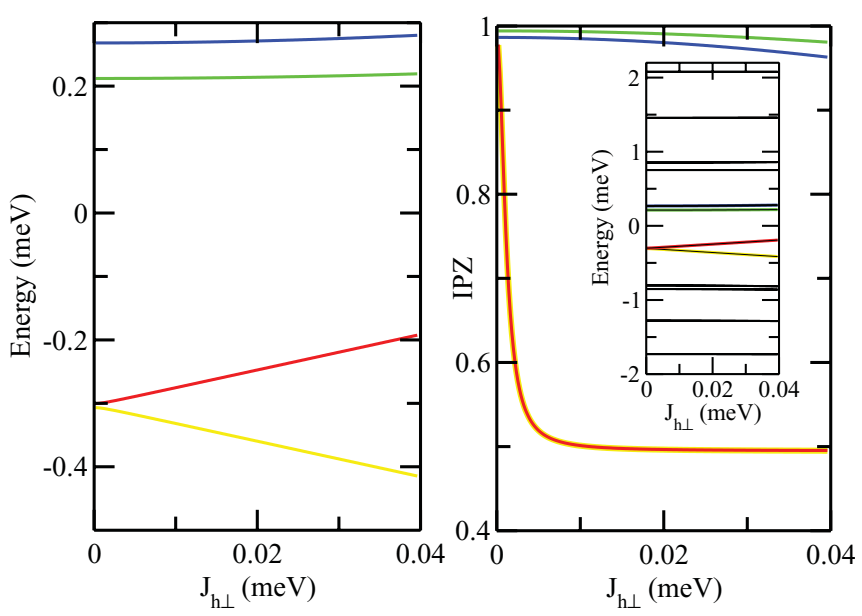

FIG. 2. (Color online) Left panel: Evolution of the exciton levels as a function of the LH-HH mixing parameter $J_{h \perp}$. Right panel: Evolution of the IPR as a function of the LH-HH mixing parameter. The inset presents the evolution of the energy for all the 24 exciton levels.

results in the observation of more than six peaks in the PL. This can be understood as follows. The spin-flip part of the hole-Mn interaction couples the bright exciton $\left|+1, M_{z}\right\rangle$ to the dark exciton $\left|-2, M_{z}+1\right\rangle$. Thus a state with dominantly dark character $\left|-2, M_{z}+1\right\rangle$ and energy given, to first order, by that of the dark exciton, has a small but finite probability of emitting a photon through its bright component, via a Mn-hole coherent spin flip. Thus PL is seen at transition energy of the dark exciton. Reversely, nominally bright excitons lose optical weight due to their coupling to the dark sector. Importantly, the emission of a photon from a dark exciton with dominant Mn spin component $M_{z}$ entails carrier-Mn spin exchange, so that the ground state has $M_{z} \pm 1$.

According to previous theory work ${ }^{18}$ the rate for the emission of a circularly polarized photon from the exciton state $\Psi_{n}$ to the ground state $\phi_{m}$ reads

$$
\Gamma_{n, m}^{ \pm}=\Gamma_{0}\left|\sum_{M_{z}} \phi_{m}\left(M_{z}\right) \Psi_{n}^{*}\left(M_{z}, X= \pm 1\right)\right|^{2},
$$

where

$$
\Gamma_{0} \equiv \frac{3 \omega^{3} d_{c v}^{2}}{4 \pi \epsilon \hbar c^{3}}
$$

is the recombination rate of the bare exciton, $\omega$ is the frequency associated to the energy difference between the exciton state $n$ and the ground state $m, c$ is the speed of light, $\epsilon$ is the dielectric constant of the material, $d_{c v}$ is the dipole matrix element. From the experiments, we infer $\Gamma_{0}=0.5 \mathrm{~ns}^{-1}$

In the absence of spin-flip terms, the matrix $\Gamma_{n, m}^{ \pm}$would have only nonzero elements for $n=\left|X= \pm 1, M_{z}\right\rangle$ states connected to $m=M_{z}$ states. The presence of spin-flip terms in the Hamiltonian enables the recombination from exciton states with the dominant dark component. In Fig. 3 we represent the matrix elements $\Gamma_{n, m}^{ \pm} / \Gamma_{0}$ for $j_{e h}=-0.73 \mathrm{meV}$, $j_{h}=0.36 \mathrm{meV}, j_{e}=-0.09 \mathrm{meV}, j_{h \perp}=0.036 \mathrm{meV}, D=$ $0.01 \mathrm{meV}$, and $E=0 \mathrm{meV}$. It is apparent that the recombination rates from the dark states are, at least, two times smaller

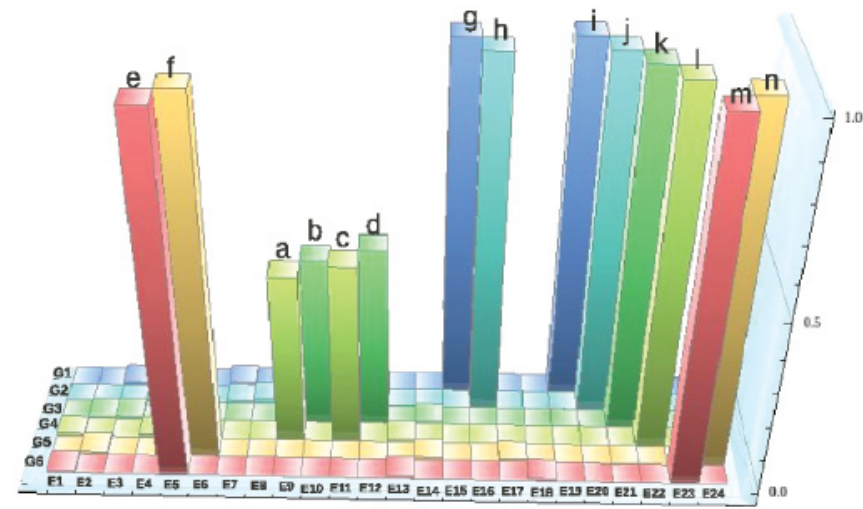

FIG. 3. (Color online) Recombination rates of the excitons levels in a Mn-doped quantum dot $\Gamma_{i} / \Gamma_{0}: a:\left|+2,+\frac{1}{2}\right\rangle \rightarrow\left|+\frac{3}{2}\right\rangle$, $b:\left|-2,-\frac{1}{2}\right\rangle \rightarrow\left|-\frac{3}{2}\right\rangle, c:\left|-1,+\frac{3}{2}\right\rangle \rightarrow\left|+\frac{3}{2}\right\rangle, d:\left|+1,-\frac{3}{2}\right\rangle \rightarrow\left|-\frac{3}{2}\right\rangle$, states from e to $\mathrm{n}$ are bright excitons. Other states are mainly dark excitons with a small bright component.

( $\mathrm{a}$ and $\mathrm{b}$ ) than those of the bright states. For $\Gamma_{0}=0.4 \mathrm{~ns}^{-1}$, the lifetime of the dark excitons ( $a$ and $b$ ) are in the range of 3 ns. Thus this provides a quite efficient Mn spin-relaxation mechanism, provided that a dark exciton is present in the quantum dot.

The recombination rate matrix, together with the nonequilibrium occupation of the exciton states, $P_{n}$, determines the PL spectrum ${ }^{18}$ for \pm circular polarization:

$$
\mathcal{I}_{\mathrm{PL}}^{ \pm}(\omega)=\sum_{n, m,} P_{n} \Gamma_{n, m}^{ \pm} \delta\left(\hbar \omega-E_{n}-E_{m}\right) .
$$

In a typical PL spectrum, ${ }^{7}$ the dark peaks are, at most, two times smaller than the bright peaks. Since $\Gamma_{n, m}^{ \pm}$is at least two times smaller for dark states, this implies a larger occupation of the dark states. Thus we can infer a transfer from the optical ground state to the dark exciton states, via the bright exciton states. This transfer requires an incoherent spin flip of either the electron or hole. Below we show that phonon induced hole-spin relaxation provides the most efficient channel for this bright to dark conversion.

\section{Mn SPIN RELAXATION DUE TO SPIN-PHONON COUPLING}

In this section we discuss the Mn spin relaxation in the absence of excitons. In the absence of carriers and given the fact that Mn-Mn distance is comparable to the dotdot distance (100 $\mathrm{nm}$ for a dot and Mn density of about $10^{10} \mathrm{~cm}^{-2}$ ), which makes direct superexchange negligible, the Mn-phonon coupling should be the dominant, albeit small, Mn spin-relaxation mechanism. Transverse phonons induce local rotations of the lattice. Since the crystal field, together with spin-orbit coupling, determines the Mn magnetocrystalline anisotropy, the phonon induced lattice rotation ${ }^{40}$ acts as a stochastic torque on the Mn spin, resulting in spin relaxation.

The atomic displacement at point $\vec{r}$ in the crystal is expressed in terms of the phonon operators with wave vector 
$\vec{q}$, polarization mode $\lambda=T 1, T 2, L$, frequency $\omega_{\lambda}(\vec{q})$, and polarization vector $\vec{e}_{\lambda}(\vec{q}),{ }^{37}$

$$
\vec{u}(\vec{r})=\sum_{\vec{q}, \lambda} \mathcal{U}_{\lambda}(\vec{q}) \vec{e}_{\lambda}(\vec{q})\left(b_{\vec{q}, \lambda}^{\dagger}+b_{-\vec{q}, \lambda}\right) e^{i \vec{q} \cdot \vec{r}},
$$

where

$$
\mathcal{U}_{\lambda}(\vec{q})=\sqrt{\frac{\hbar}{2 \rho \omega_{\lambda}(\vec{q}) V}},
$$

and $V$ and $\rho$ are the volume of the crystal and the mass density, respectively. In a zinc-blende structure there are two transverse acoustic (TA) phonon branches and one longitudinal acoustic branch (LA). Following Woods ${ }^{31}$ we have

$$
\begin{gathered}
\vec{e}_{T A 1}=\frac{1}{q q_{\perp}}\left(q_{x} q_{z}, q_{y} q_{z},-q_{\perp}^{2}\right), \\
\vec{e}_{T A 2}=\frac{1}{q_{\perp}}\left(q_{y},-q_{x}, 0\right),
\end{gathered}
$$

where $q \equiv|\vec{q}|$ and $q_{\perp}=\sqrt{q_{x}^{2}+q_{y}^{2}}$. These vectors satisfy $\vec{q}$. $\vec{e}_{T A i}=0$, and $\vec{e}_{T A i} \cdot \vec{e}_{T A j}=\delta_{i j}$ The longitudinal mode has $\vec{e}_{L A}=\frac{1}{q} \vec{q}$. The lattice rotation vector is given by ${ }^{40}$

$$
\overrightarrow{\delta \Phi}(\vec{r})=\vec{\nabla} \times \vec{u}(\vec{r})
$$

so that only the transverse modes contribute. Within this picture, the Mn spin-phonon coupling can be written as ${ }^{40}$

$$
\mathcal{V}_{\mathrm{M}-\mathrm{ph}}=i\left[\mathcal{H}_{0}, \vec{M}\right] \cdot \overrightarrow{\delta \Phi}\left(\vec{r}_{\mathrm{Mn}}\right)
$$

Without loss of generality we can set the Mn position as the origin, $\vec{r}_{\mathrm{Mn}}=0$. Equation (50) couples the Mn spin to a reservoir of phonons whose noninteracting Hamiltonian is

$$
\mathcal{H}_{\mathrm{ph}}=\sum_{\vec{q}, \lambda} \hbar \omega_{\lambda}(\vec{q}) b_{\vec{q}, \lambda}^{\dagger} b_{\vec{q}, \lambda} .
$$

Within the standard system plus reservoir master equation approach, we have derived the scattering rate from a state $n$ to a state $n^{\prime}$, both eigenstates of the single Mn Hamiltonian $\mathcal{H}_{0}$, due the emission of a phonon. In order to use a general result for that rate (B7), derived in Appendix B, we need to express the spin-phonon coupling (50) using the same notation as in Eq. (B1):

$$
\mathcal{V}_{\vec{q}, \lambda}^{n, n^{\prime}}=i^{2} \mathcal{U}_{\lambda}(\vec{q}) \vec{f}_{n, n^{\prime}} \cdot\left[\vec{q} \times \vec{e}_{\lambda}(\vec{q})\right]
$$

where

$$
\vec{f}_{n, n^{\prime}} \equiv\left\langle n\left|\left[\mathcal{H}_{0}, \vec{M}\right]\right| n^{\prime}\right\rangle .
$$

We compute now the scattering rate due to a single phonon emission assuming three-dimensional phonons described above. The rate reads

$$
\Gamma_{n \rightarrow n^{\prime}}=\frac{|\Delta|^{3}}{12 \pi \rho \hbar^{4} c^{5}}\left[n_{B}(\Delta)+1\right] \sum_{b, b^{\prime}=x, y, z} f_{n^{\prime}, n}^{b}\left(f_{n^{\prime}, n}^{b^{\prime}}\right)^{*},
$$

where $c=1.79 \mathrm{~km} \mathrm{~s}^{-1}$ is the CdTe speed of sound, ${ }^{41} \rho=$ $5870 \mathrm{~kg} \mathrm{~m}^{-3}$ is the mass density of the CdTe unit cell ${ }^{42}$ and $n_{B}(\Delta) \equiv \frac{1}{e^{\beta|\Delta|}-1}$. The $|\Delta|^{3}$ factor comes from the dependence of the phonon density of states on the energy.

\section{A. Mn spin relaxation in the optical ground state}

We now discuss the relaxation of the Mn electronic spin due to spin-phonon coupling without an exciton in the quantum dot. According to our experimental results, ${ }^{5,7}$ the Mn spinrelaxation time in our samples is at least $5 \mu \mathrm{s}$.

If we take $E=0$, the transition rate between the excited states $\left|\phi_{n}\right\rangle=\left|M_{z}=+\frac{5}{2}\right\rangle$ and $\left|\phi_{n^{\prime}}\right\rangle=\left|M_{z}=+\frac{3}{2}\right\rangle$, via a phonon emission, is given by

$$
\Gamma_{n \rightarrow n^{\prime}}=\frac{640|D|^{5}}{3 \pi \rho \hbar^{4} c^{5}}\left[n_{B}(\Delta)+1\right] .
$$

The dependence on $D^{5}$ comes both from the density of states of phonons $\rho \propto \omega^{3}$ and the square of the Mn phonon coupling, which is proportional to the anisotropy, and gives the additional $D^{2}$ factor. Whereas the uniaxial anisotropy of $\mathrm{Mn}$ in $\mathrm{CdTe}$ quantum wells has been determined by EPR, ${ }^{33}$ the actual value for $\mathrm{Mn}$ in quantum dots is not known and cannot be measured directly from single exciton spectroscopy of neutral dots. Therefore in Fig. 4 we plot the lifetime for the transition of the Mn spin from $\frac{5}{2}$ to $\frac{3}{2}$, due to a phonon emission, as a function of $D$. We take $D$ in a range around the value reported for CdTe:Mn epilayers, $D=12 \mu \mathrm{eV} .{ }^{33}$ We find that the spin lifetime of $\mathrm{Mn}$ in the optical ground state can be very large. Even for $D=20 \mu \mathrm{eV}$ the Mn spin lifetime is in the range of $0.1 \mathrm{~s}$, well above the lower limit for the Mn spin relaxation reported experimentally. ${ }^{5,7}$ Whereas we cannot rule out completely the Mn spin lifetimes that long, there are other spin-relaxation mechanisms that might be more efficient that the Mn-phonon coupling considered above, like the coupling of the Mn electronic spin to nuclear spins of Mn and the host atoms. ${ }^{5}$

This model can be used also in the case limit where the $D$ is much smaller and the energy splittings are given by the applied field $B$. In that case one could expect spinrelaxation rates proportional to $B^{5}$. This $B^{5}$ behavior has been observed ${ }^{43}$ in very diluted $(x=0.002)$ bulk $\mathrm{Cd}_{1-x} \mathrm{Mn}_{x} \mathrm{Te}$ at very high magnetic fields $B>10 \mathrm{~T}$, giving qualitative support
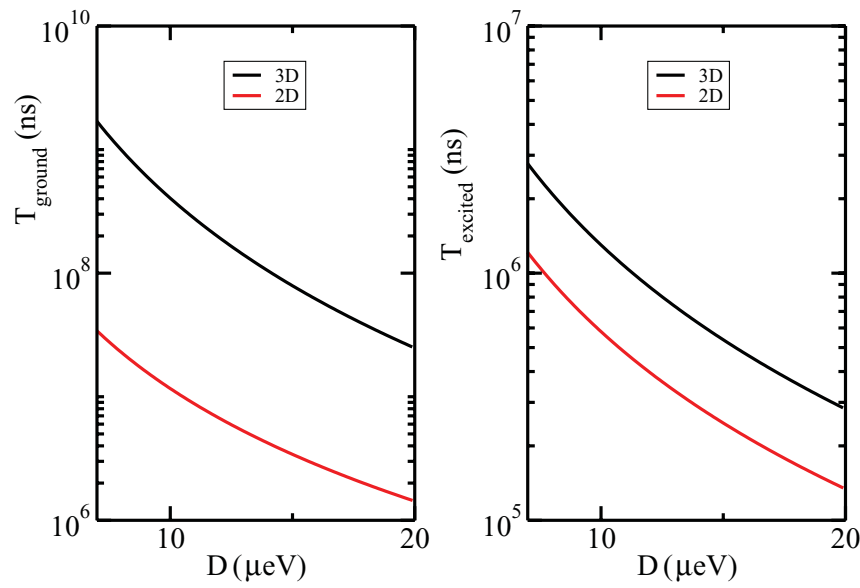

FIG. 4. (Color online) Left panel: Lifetime of the $\left(+\frac{5}{2}\right.$ to $\left.+\frac{3}{2}\right)$ transition in the optical ground state at zero field as a function of the magnetic anisotropy energy splitting $D$. Right panel: Lifetime of the same transition in the presence of a +1 exciton for different values of $D$. The rates are calculated for a three-dimensional (3D) and a two-dimensional (2D) density of states of acoustic phonons. 
to the notion that phonon induced relaxation is the dominant mechanism in the dilute limit. Quantitatively, though, the observed relaxation rate at $B=20 \mathrm{~T}$ was $\Gamma \simeq 10^{6} \mathrm{~s}^{-1}$, which corresponds to a lifetime of $T_{1} \simeq 1 \mu \mathrm{s}$ for a splitting of $1 \mathrm{meV}$, much larger than what we would extrapolate from Fig. 4.

Since part of the $\Delta^{5}$ scaling arises from the $\omega^{3}$ scaling of the phonon density of states, we have explored the possibility that phonons localized in the wetting layer could be more efficient in relaxing the Mn spin. For that matter we have considered a toy model of two-dimensional phonons confined in a slab of thickness $W=2 \mathrm{~nm}$. The resulting Mn spin-relaxation rate for those reads

$$
\Gamma_{n \rightarrow n^{\prime}}=\frac{\Delta^{2}}{16 \hbar^{3} c^{4} \rho W}\left[n_{B}(\Delta)+1\right] \sum_{b, b^{\prime}=x, y, z} A_{b, b^{\prime}} f_{n^{\prime}, n}^{b}\left(f_{n^{\prime}, n}^{b^{\prime}}\right)^{*},
$$

where $W$ is the width of the sample and $A$ is a diagonal matrix with $A_{x x}=1, A_{y y}=1, A_{z z}=2$.

In Fig. 4 we plot the associated spin lifetime in this case, taking $W=2 \mathrm{~nm}$ and show how it is at least 100 shorter than for $3 \mathrm{D}$ phonons, but still we would have $T_{1} \simeq 1 \mathrm{~ms}$ for $D=20 \mu \mathrm{eV}$.

\section{B. Mn spin relaxation in the presence of an exciton}

Here we discuss how the Mn spin relaxation due to Mnphonon coupling is modified when an exciton is interacting with the Mn. The Mn-phonon coupling is still given by Hamiltonian (50), with $\mathcal{H}_{0}$ given by Eq. (1). We assume that the only effect of the exciton on the $\mathrm{Mn}$ is to change the energy spectrum and mix the spin-wave functions, giving rise to larger spin relaxation rates, due to the larger exchange induced energy splittings.

In the presence of the exciton, the Mn-phonon coupling results in transitions between different exciton-Mn spin states, $n$ and $n^{\prime}$. As we did in the case of the Mn without excitons, we need to express the spin-phonon coupling (50) using the same notation as in Eq. (B1).

For that matter we define the matrix elements

$$
\begin{aligned}
\vec{F}_{n, n^{\prime}} & \equiv\left\langle\Psi_{n}\left|\left[\mathcal{H}_{0}, \vec{M}\right]\right| \Psi_{n}^{\prime}\right\rangle \\
& =\sum_{X, M_{z}, M_{z}^{\prime}} \Psi_{n}(X, M)^{*} \Psi_{n}\left(X, M^{\prime}\right)^{*} \vec{f}_{M, M^{\prime}},
\end{aligned}
$$

where

$$
\vec{f}_{M, M^{\prime}} \equiv\left\langle M\left|\left[\mathcal{H}_{0}, \vec{M}\right]\right| M^{\prime}\right\rangle
$$

$M$ and $M^{\prime}$ stand for eigenstates of the Mn spin operator $M_{z}$. Thus, in the exciton-Mn spin states basis, the Mn-phonon coupling reads

$$
\mathcal{V}_{\vec{q}, \lambda}^{n, n^{\prime}}=i^{2} \mathcal{U}_{\lambda}(\vec{q}) \vec{F}_{n, n^{\prime}}(M) \cdot\left[\vec{q} \times \vec{e}_{\lambda}(\vec{q})\right]
$$

Notice how if we neglect the spin mixing of the exciton states we have $\vec{F}_{n, n^{\prime}}=\vec{f}_{M, M^{\prime}}$ and the only difference in the scattering rates arises from the larger energy splittings in the presence of the exciton.
Using Eq. (B7) for the phonon induced spin-relaxation rate, and in analogy with Eq. (54), we write

$$
\Gamma_{n \rightarrow n^{\prime}}=\frac{|\Delta|^{3}}{12 \pi \rho \hbar^{4} c^{5}}\left[n_{B}(\Delta)+1\right] \sum_{b, b^{\prime}=x, y, z} F_{n, n^{\prime}}^{b}\left(F_{n, n^{\prime}}^{b^{\prime}}\right)^{*}
$$

In Fig. 4 we see how Mn-phonon spin relaxation is much faster in the presence of the exciton. Ignoring the difference arising from the spin mixing, we can write the ratio of the rates as

$$
\frac{\Gamma_{n \rightarrow n^{\prime}}(X)}{\Gamma_{n \rightarrow n^{\prime}}(G)}=\left(\frac{\Delta_{X}}{\Delta_{G}}\right)^{3}
$$

The energy splitting associated to the $\frac{5}{2}$ to $\frac{3}{2}$ spin flip in the ground state is $4 D$. In the presence of the exciton the energy splitting of the same transition would be $4 D+j_{h}-j_{e}$. If we take $D=12 \mu \mathrm{eV}, j_{h}=360 \mu \mathrm{eV}$, and $j_{e}=-90 \mu \mathrm{eV}$ the ratio yields $\approx 10^{3}$. From the experimental side we know that $T_{1 G}>5 \mu \mathrm{s}$ and, in the presence of the exciton $T_{1} \simeq 50 \mathrm{~ns}$. Thus the ratio could be accounted for by this mechanism. However, in order to have $T_{1 G}=5 \mu \mathrm{s}$ we would need to assume an unrealistically large value for $D$. Thus we think that another spin-relaxation mechanism must be operative in the system when the exciton is in the dot, which makes it possible to control the spin of the $\mathrm{Mn}$ in a time scale of $50 \mathrm{~ns}$. In the next sections we discuss the hole spin relaxation due to phonons as the mechanism that, combined with Mn-carrier exchange, yields a quick Mn spin relaxation in the presence of the exciton.

\section{HOLE SPIN RELAXATION IN NONMAGNETIC DOTS}

\section{A. Hole-phonon coupling}

We now consider the relaxation of the hole spin due to hole-phonon coupling. We consider first the case of undoped quantum dots. The coupling of the spin of the hole to phonons can be understood extending the Bir-Pikus Hamiltonian to the case of inhomogeneous strain associated to lattice vibrations:

$$
\epsilon_{i j}(\vec{r}) \equiv \frac{1}{2}\left(\frac{\partial u_{i}}{\partial r_{j}}+\frac{\partial u_{j}}{\partial r_{i}}\right)
$$

It is convenient to write the strain tensor field as

$$
\epsilon_{i j}(\vec{r})=\sum_{\vec{q}} e^{i \vec{q} \cdot \vec{r}} \epsilon_{i j}(\vec{q})
$$

so that we write

$$
\epsilon_{i j}(\vec{q})=\frac{1}{2} \sum_{\lambda} \mathcal{U}_{\lambda}(\vec{q})\left(b_{\vec{q}, \lambda}^{\dagger}+b_{-\vec{q}, \lambda}\right)\left[q_{j} e_{\lambda}^{i}(\vec{q})+q_{i} e_{\lambda}^{j}(\vec{q})\right] .
$$

We consider the coupling of the ground-state doublet, formed by states $\Uparrow$ and $\Downarrow$, to the phonon reservoir. ${ }^{31,44,45}$ The effective hole-phonon Hamiltonian is obtained by projecting 
the BP Hamiltonian onto this subspace:

$$
\mathcal{V}_{\mathrm{h} \text {-phonon }}=\sum_{i j, \vec{q} \sigma_{h}, \sigma_{h}^{\prime}} I_{i j}^{\sigma_{h}, \sigma_{h}^{\prime}}(\vec{q})\left|\sigma_{h}\right\rangle\left\langle\sigma_{h}^{\prime}\right| \epsilon_{i j}(\vec{q}) .
$$

Here $\left|\sigma_{h}\right\rangle$ denotes the quantum dot state defined in Eq. (20) and the coupling constant reads

$$
I_{i j}^{\sigma_{h}, \sigma_{h}^{\prime}}(\vec{q}) \equiv \sum_{j_{z}, j_{z}^{\prime}} V_{i j} \mathcal{C}_{h}^{*}\left(j_{z}\right) \mathcal{C}_{h^{\prime}}\left(j_{z}^{\prime}\right)\left\langle j_{z}\left|J_{i} J_{j}\right| j_{z}^{\prime}\right\rangle I_{\vec{q}},
$$

where $I_{\vec{q}}=\int\left|\psi_{0}(\vec{r})\right|^{2} e^{i \vec{q} \cdot \vec{r}} d \vec{r}$. Hamiltonian (65) shows how the absorption or emission of a phonon can induce a transition between the two quantum dot hole states, $\Uparrow$ and $\Downarrow$.

We now calculate the time scale for the spin relaxation of a single hole in a nonmagnetic dot under the influence of an applied magnetic field so that the hole ground-state doublet is split in energy. In order to compute the transition rate for decay of the hole from the excited to the ground state we use again the general equation (B7). For that matter, we express the hole-spin coupling (65) as

$$
\mathcal{V}_{\text {h-phonon }}=\sum_{\vec{q}, \lambda \sigma_{h}, \sigma_{h}^{\prime}} \mathcal{V}_{\vec{q}, \lambda}^{\sigma_{h}, \sigma_{h}^{\prime}}\left|\sigma_{h}\right\rangle\left\langle\sigma_{h}^{\prime}\right|\left(b_{\lambda q}^{\dagger}+b_{\lambda,-q}\right),
$$

where

$$
\mathcal{V}_{\vec{q}, \lambda}^{\sigma_{h}, \sigma_{h}^{\prime}}=\frac{i}{2} \sum_{i, j} I_{i j}^{\sigma_{h}, \sigma_{h}^{\prime}}(\vec{q}) \mathcal{U}_{\lambda}(\vec{q})\left[q_{i} e_{\lambda}^{j}(\vec{q})+q_{j} e_{\lambda}^{i}(\vec{q})\right] .
$$

\section{B. Calculation of hole spin-flip rates with simple model}

In order to illustrate the physics of the phonon-driven hole spin relaxation we consider the case of a single hole in a nonmagnetic dot under the influence of an applied magnetic field. For that matter, we compute the Hamiltonian (68) using the wave functions from the simple model of confined holes defined in Eq. (12). We focus on the nondiagonal terms in the hole spin index, i.e., the terms that result in scattering from $\Uparrow$ to $\Downarrow$ due to phonon emission.

Importantly, the BP Hamiltonian couples hole states that differ in, at most, two units of $J_{z}$. Thus in the absence of LH-HH mixing, the BP Hamiltonian does not couple directly the $\Uparrow$ and $\Downarrow$ states. Transitions between $\Uparrow$ and $\Downarrow$ states, as defined in Eq. (20), are only possible, through one phonon process, through the $\epsilon_{y z}\left(J_{y} J_{z}+J_{z} J_{y}\right)$ and $\epsilon_{z x}\left(J_{z} J_{x}+J_{x} J_{z}\right)$ terms in the Hamiltonian. After a straightforward calculation we obtain

$$
\mathcal{V}_{\vec{q}, \lambda}^{\Uparrow, \Downarrow}=\frac{i}{2} \sqrt{3} d \sin \left(\frac{\theta_{1}-\theta_{2}}{2}\right)\left[\epsilon_{y z}(\vec{q})-i \epsilon_{z x}(\vec{q})\right] .
$$

The important role played by the LH-HH mixing $\theta_{1,2}$ is apparent. Using Eq. (B7) it is quite straightforward to compute the rate for the three phonon branches. They are all proportional to

$$
\Gamma_{\Uparrow \rightarrow \Downarrow}^{0}=\frac{1}{18 \pi} D_{u^{\prime}}^{2} \sin ^{2}\left(\frac{\theta_{1}-\theta_{2}}{2}\right) \frac{\Delta^{3}}{\rho \hbar^{4} c^{5}}
$$

with coefficients $\frac{7}{5}, 1$, and $\frac{8}{5}$ for the $T A 1, T A 2$, and $L$ modes, respectively. Here, $D_{u^{\prime}}$ stands for the deformation potential of Kleiner and Roth, ${ }^{46}$ following Ref. 36, $D_{u^{\prime}}=-\frac{3 \sqrt{3} d}{2}, \rho$ stands for the mass density of CdTe, $c$ stands for its transverse speed
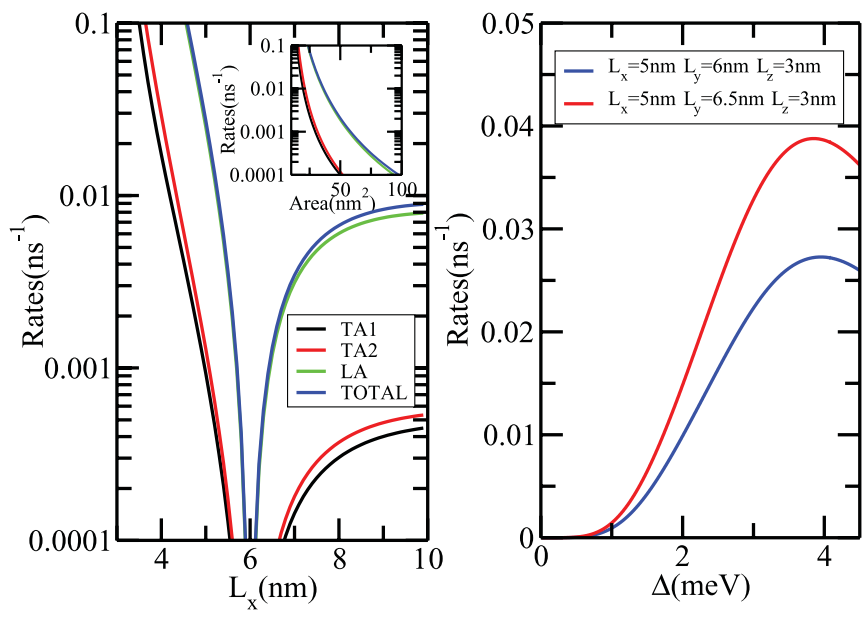

FIG. 5. (Color online) Left panel: Hole spin-flip rate as a function of the dot size and shape. $L_{y}$ is fixed at $6 \mathrm{~nm}$. In the inset the ratio $L_{y} / L_{x}$ is fixed at 1.2 so the shape of the dot are fixed, only the size of the dot changes. One can see that the hole spin-flip rate is a size sensitive quantum quantity, the rate is a semiexponential function of the size of the dot. Right panel: Hole spin-flip rate as a function of the energy splitting for two different values of the quantum dot anisotropy, i.e., LH-HH mixing.

of sound, and $\Delta$ stands for the energy splitting between the $\Uparrow$ and $\Downarrow$ states, which is proportional to the external magnetic field $B$. In Fig. 5 we plot the rates $\Gamma_{T A 1}, \Gamma_{T A 2}, \Gamma_{L}$, as well as their sum as function of the dot size (left panel) and as a function of the energy splitting between the initial and final hole state, $\Delta$ (right panel). We see how hole spin-relaxation rates can be in the range of $\Gamma \simeq 1 /(40 \mathrm{~ns})$.

The results of Fig. 5 suggest that for sufficiently high $\Delta$, as those provided by the Mn-hole exchange, the hole spin can relax in a time scale of $30 \mathrm{~ns}$. These numbers are in the same range as those obtained by Woods et al.${ }^{31}$ As we discuss in the next section, these spin flips, together with Mn-carrier exchange, can also induce $\mathrm{Mn}$ spin relaxation in a time scale much shorter than the one due to Mn-phonon coupling only. Notice that the saturation of the hole spin-relaxation rate as the energy splitting is increased is related to the phonon wavelength becoming smaller than the dot size, leading to a suppression of the matrix element $I_{\vec{q}} \cdot{ }^{47}$

Importantly, the rate is finite only if $\theta_{1}-\theta_{2} \neq 0$, which is the case in the presence of an applied magnetic field. This indicates that, within the simple model of Eq. (12), the nondiagonal terms in the hole-phonon Hamiltonian (65) vanishes identically. Similar cancellation of the spin relaxation at zero field occur in other models and might be a general result. ${ }^{48}$ The exchange coupling of the hole to either Mn or electron spin are expected to have a similar effect on the hole wave functions, and thereby on the phonon induced hole spin relaxation, as the magnetic field. However, this effect is only captured if the system is described with the complete hole basis, i.e., without removing from the Hilbert space the two high-energy hole states. It is worth pointing out that hole spin relaxation is thereby very different in an electron (or a $\mathrm{Mn}$ atom) are present in the dot. In the presence of an electron, zero-field hole spin relaxation can take place. ${ }^{44}$ 


\section{SPIN RELAXATION IN MAGNETIC DOTS DUE TO HOLE-PHONON COUPLING}

The results of the previous sections indicate that, because of their coupling to phonons, the hole spin lifetime in a nonmagnetic dot is much shorter than the Mn spin lifetime. Here we explore the consequences of this phonon-driven hole spin relaxation for the single exciton states in a dot doped with one magnetic atom. The leading process results in a Mn spin conserving decay from the bright exciton to the dark exciton state, via hole-spin flip in a time scale in the 10-ns range. Combined with the optical recombination of the dark state, made possible via Mn-hole or Mn-electron spin flip, this provides a pathway for exciton induced Mn spin relaxation in a time scale under $100 \mathrm{~ns}$, as observed experimentally. ${ }^{5-7}$

We also explore the scattering between two bright states enabled by the combination of phonon induced hole spin relaxation and Mn-carrier exchange. The lifetimes of these processes is in the range of $10^{3} \mathrm{~ns}$ and higher, and therefore they are probably not determinant for the optical orientation of the Mn spin in the submicrosecond scale.

\section{A. Exciton-phonon coupling in magnetic dots}

The Hamiltonian that couples the exciton states $\Psi_{n}$ to the phonons is derived by projecting the hole-phonon coupling Hamiltonian (65) onto the exciton states (40). The result reads

$$
\mathcal{V}_{\mathrm{X} \text {-phon }}=\sum_{n, n^{\prime} \vec{q} \lambda}\left|\Psi_{n}\right\rangle\left\langle\Psi_{n^{\prime}}\right| \mathcal{V}_{\vec{q}, \lambda}^{n, n^{\prime}}\left(b_{\lambda q}^{\dagger}+b_{\lambda,-q}\right),
$$

where

$$
\mathcal{V}_{\vec{q}, \lambda}^{n, n^{\prime}}=\sum_{M_{z}, \sigma_{e}, \sigma_{h}, \sigma_{h}^{\prime}} \mathcal{V}_{\vec{q}, \lambda}^{\sigma_{h}, \sigma_{h}^{\prime}} \Psi_{n}\left(M_{z}, X\right) \Psi_{n^{\prime}}^{*}\left(M_{z}, X^{\prime}\right),
$$

where $X=\left(\sigma_{e}, \sigma_{h}\right)$ and $X^{\prime}=\left(\sigma_{e}, \sigma_{h}^{\prime}\right)$ (same electron spin) and $\mathcal{V}_{\vec{q}, \lambda}^{\sigma_{h}, \sigma_{h}^{\prime}}$ is given by Eq. (68).

\section{B. Qualitative description of the spin-relaxation processes}

In order to describe qualitatively the variety of different processes accounted for by Hamiltonian (71) it is convenient to consider an initial state $\psi_{n}$ as a linear combination of a dominant component $|M\rangle|\downarrow\rangle_{e}|\uparrow\rangle_{h}$ plus a minor contribution of two dark components, which arise from the coherent exchange of the Mn with either the electron or the hole:

$$
\begin{aligned}
\left|\psi_{n}\right\rangle= & |M\rangle|\downarrow\rangle_{e}|\Uparrow\rangle_{h}+\epsilon_{e}|M-1\rangle|\uparrow\rangle_{e}|\Uparrow\rangle_{h} \\
& +\epsilon_{h}|M+1\rangle|\downarrow\rangle_{e}|\Downarrow\rangle_{h},
\end{aligned}
$$

where $\epsilon_{e} \propto j_{e} / j_{e h}$ and $\epsilon_{h} \propto j_{h} / j_{e h}$ are small dimensionless coefficients that can be obtained doing perturbation theory.

Depending on the elementary process that takes place, there are several possible final states:

(1) Hole spin relaxation. In this case the final state would be dominantly a dark exciton whose wave function reads

$$
\left|\psi_{n^{\prime}}\right\rangle=|M\rangle|\downarrow\rangle_{e}|\Downarrow\rangle_{h}+O(\epsilon)
$$

and the scattering rate $\Gamma_{0}$ would be proportional to $\left|I^{\Uparrow, \Downarrow}\right|^{2}$. This is process II in Fig. 6.

(2) Hole spin relaxation plus coherent hole-Mn spin flip. This is process III in Fig. 6. This can be realized through two

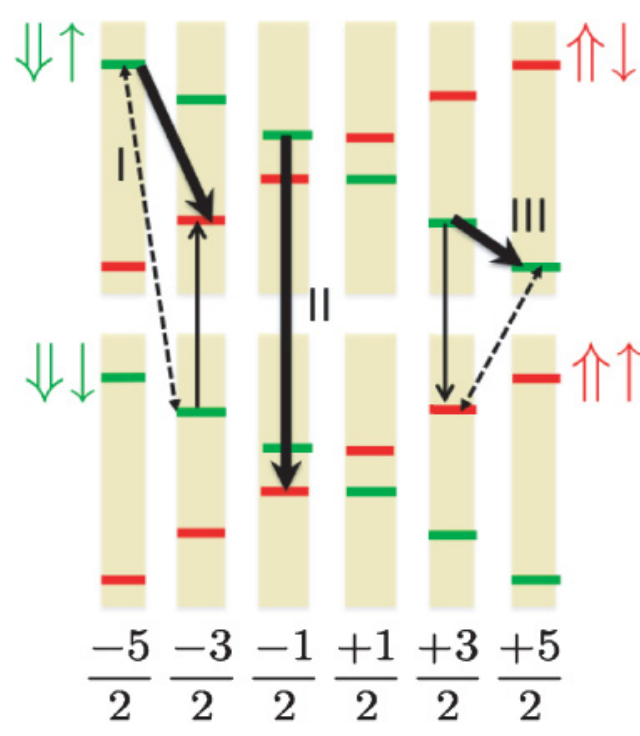

FIG. 6. (Color online) Scheme of the Mn spin-flip channels due to the combined action of hole-phonon coupling and carrier-Mn exchange.

dominant channels. An incoherent hole spin flip will couple the dominant component of the initial state, $|M\rangle|\downarrow\rangle_{e}|\Uparrow\rangle_{h}$, with a secondary component $|M\rangle|\downarrow\rangle_{e}|\Downarrow\rangle_{h}$ of the final state,

$$
\left|\psi_{n^{\prime}}\right\rangle=|M-1\rangle|\downarrow\rangle_{e}|\Uparrow\rangle_{h}+\epsilon_{h}|M\rangle|\downarrow\rangle_{e}|\Downarrow\rangle_{h}+O\left(\epsilon_{e}\right) .
$$

In this case the final state is a bright exciton in the same branch +1 as the initial state but the Mn component goes from $M$ to $M-1$. The second channel comes from the hole spin flip of the minority dark component of the initial state, $\epsilon_{h}|M+1\rangle|\downarrow\rangle_{e}|\Downarrow\rangle_{h}$ which decays into the majority component of the final state,

$$
\left|\psi_{n^{\prime}}\right\rangle=|M+1\rangle|\downarrow\rangle_{e}|\Uparrow\rangle_{h}+O\left(\epsilon_{e, h}\right) .
$$

Thus in this second case a hole spin flips due to phonons plus a coherent Mn-electron spin flip connects the $X=+1, M$ initial state to the $X=-1, M+1$ state. Thus both the initial and final state in this process are the same as in the first channel; the rates for each would be proportional to $\epsilon_{h}^{2} \Gamma_{0}$, but the decay pathways are different, and interferences are expected.

(3) Hole spin relaxation plus coherent electron-Mn spin flip. This is process I in Fig. 6. As in the previous case, there are two channels for this type of process. In the first channel, the majority component of the initial state decays into a final state given by

$$
\left|\psi_{n^{\prime}}\right\rangle=|M-1\rangle|\uparrow\rangle_{e}|\Downarrow\rangle_{h}+\epsilon_{e}^{\prime}|M\rangle|\downarrow\rangle_{e}|\Downarrow\rangle_{h} .
$$

The incoherent hole spin flip connects the initial state (73) to the final state (77) through the minority component $|M\rangle|\downarrow\rangle_{e}|\downarrow\rangle_{h}$ of the latter. The second channel comes from the hole spin flip of the minority dark component of the initial state, $\epsilon_{e}|M-1\rangle|\uparrow\rangle_{e}|\Uparrow\rangle_{h}$, which decays into the majority component of the final state,

$$
\left|\psi_{n^{\prime}}\right\rangle=|M-1\rangle|\uparrow\rangle_{e}|\Downarrow\rangle_{h}+O\left(\epsilon_{e, h}\right) .
$$

Thus a hole spin flips due the phonon plus a coherent Mnelectron spin flip connects the $X=+1, M$ initial state to the 
$X=-1, M-1$ state. The scattering rate of these two process scales as $\epsilon_{e}^{2} \Gamma_{0}$.

\section{Calculation of the relaxation rates}

In order to implement Eqs. (71) and (72) to compute scattering rates, we use the single-particle basis for the holes done with Eqs. (12) which leads, at finite magnetic field, to the matrix element (69) that would be incorporated into Eq. (72) to compute the rates using Eq. (B7). As discussed above, a zero-field model (12) yields a zero spin-flip matrix element in Eq. (69). This is a feature of the simple hole model rather than an intrinsic property of the system. Thus for the sake of simplicity, we compute the rates between exciton states by computing the matrix element (69) as if there was a magnetic field that yields the energy splitting between the initial and final exciton states equal to the splitting produced by the exchange interaction with the Mn spin. This approximation would not be necessary if we kept the four eigenstates of the single-hole Hamiltonian, instead of keeping only the low-energy doublet.

In the calculation of the rates we perform an additional approximation: we only consider spin-flip terms in Eq. (72) and we do exclude spin-conserving terms. The results for transition rates from the state $n$ with dominant $\left(-1,-\frac{5}{2}\right)$ to three possible final states with dominant components $\left(+2,-\frac{5}{2}\right),\left(-2,-\frac{1}{2}\right)$, and $\left(+1,-\frac{3}{2}\right)$ as a function of the spin-flip Mn-hole exchange $J_{h \perp}$ are shown in Fig. 7. The transition to the $\left(+2,-\frac{5}{2}\right)$, which only involves the irreversible spin flip of the hole via a phonon emission, is the dominant process and has a lifetime of 30 ns. The transition to the $\left(+1,-\frac{3}{2}\right)$ state requires both the hole spin flip and the Mn-hole spin flip and it is three orders of magnitude less efficient.

Thus these calculations indicate that the most likely mechanism for Mn spin orientation in the presence of an exciton combines a rapid bright to dark conversion, produced by phonon induced hole spin flip and a dark to ground transition, enabled by $\mathrm{Mn}$-carrier spin exchange and radiative recombination.
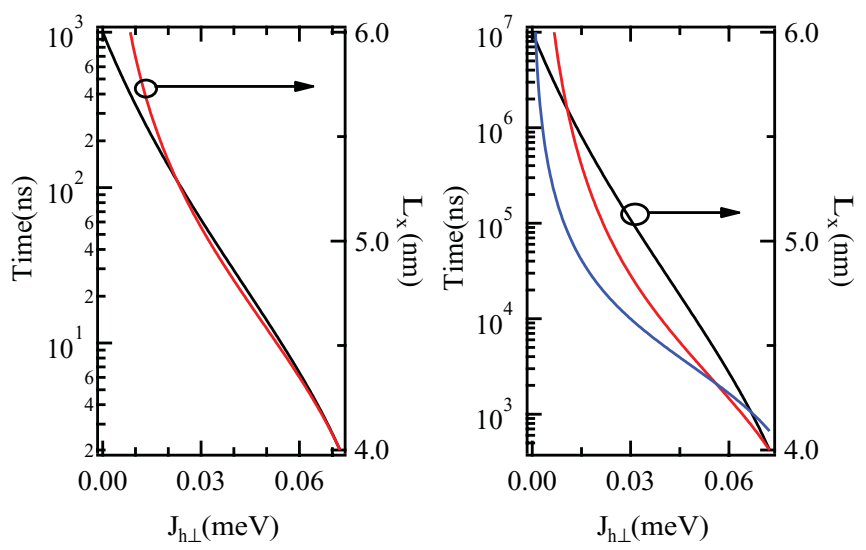

FIG. 7. (Color online) Calculated rates for the transitions between exciton states in a Mn-doped quantum dot due to hole-phonon coupling. Left panel, red line, transition from $\left|-1,-\frac{5}{2}\right\rangle$ to $\left|+2,-\frac{5}{2}\right\rangle$. Right panel, red line, transition from $\left|-1,-\frac{5}{2}\right\rangle$ to $\left|-2,-\frac{1}{2}\right\rangle$; blue line, transition from $\left|-1,-\frac{5}{2}\right\rangle$ to $\left|+1,-\frac{3}{2}\right\rangle . L_{y}$ is fixed at $6 \mathrm{~nm}$ and scanning $L_{x}$ changes the LH-HH mixing parameter $J_{h \perp}$.

\section{LASER DRIVEN SPIN DYNAMICS}

\section{A. Summary of scattering mechanisms and master equation}

The spin dynamics of a single $\mathrm{Mn}$ atom in a laser driven quantum dot is described in terms of the 24 exciton states $\Psi_{n}$ and the 6 ground states $\phi_{m}$. In the previous sections we have calculated the scattering rates of these states. They can be summarized as follows:

(1) Transitions from the $\Psi_{n}$ to the $\phi_{m}$, via photon emission [Eq. (42)]. In the case of bright excitons, this process is the quickest of all, with a typical lifetime of $0.3 \mathrm{~ns}$. In the case of dark excitons the lifetime depends on the bright/dark mixing, which is both level and dot dependent. Dark lifetime ranges from twice the one of bright excitons to 1000 times larger, i.e., between 1 and 300 ns. In any event, dark recombination involves a Mn spin flip.

(2) Transitions between different $\phi_{m}$ states, due to Mn spinphonon coupling [Eq. (54)]. The lifetimes of these transitions are, at least, $1 \mathrm{~ms}$ (see the right panel of Fig. 4).

(3) Transitions between different exciton states $\Psi_{n}$ that flip the spin of the Mn only, due to Mn-phonon coupling [Eq. (60)]. The lifetimes of these transitions are, at least, $0.1 \mathrm{~ms}$ (see the left panel of Fig. 4).

(4) Transitions between exciton states due to hole-phonon coupling [Eq. (71)]. The bright to dark transition is the quickest process with a lifetime of about $30 \mathrm{~ns}$ (see Fig. 7). Bright to bright transitions, combining hole-phonon and Mn-carrier interactions, have lifetimes in the $10-\mu$ s range.

In addition to these dissipative scattering processes, we have to consider the driving effect of the laser field, described in the semiclassical approximation. All things considered, we arrive at a master equation that describes the evolution of the occupations $p_{N}$, where $N=(n, m)$ includes states both with and without an exciton in the dot. The master equation reads

$$
\frac{d p_{N}}{d t}=\sum_{N^{\prime}} \Gamma_{N^{\prime} \rightarrow N} p_{N^{\prime}}-\sum_{N^{\prime}} \Gamma_{N \rightarrow N^{\prime}} p_{N} .
$$

Equation (79) is a system of 36 coupled differential equations that we solve by numerical iteration, starting from a thermal distribution for the initial occupation $p_{N}$. Since the temperature is larger than the energy splitting in the ground state, but much smaller than the band gap, at $t=0$ we have the six ground states with similar occupation $P_{m} \simeq 1 / 6, P_{n} \simeq 0$. As a result, the average magnetization, defined as

$$
\left\langle M_{z}\right\rangle=\sum_{m} p_{m}\left\langle\phi_{m}\left|M_{z}\right| \phi_{m}\right\rangle,
$$

is zero, at zero magnetic field, as expected.

\section{B. Optical Mn spin orientation}

Under the action of the laser, the exciton states become populated and, under the adequate pumping conditions, the average Mn magnetization $\left\langle M_{z}\right\rangle$ acquires a nonzero value. This transfer of angular momentum, known as optical Mn spin orientation, has been observed experimentally ${ }^{5}$ and predicted theoretically. $^{27}$ It results from a decrease of the Mn spin lifetime in the presence of the exciton in the dot. In that circumstance, the laser transfers population from the $M_{z}$ state to the $X, M_{z}$ state. The enhanced relaxation transfers 
population from $X, M_{z}$ to $X, M_{z}^{\prime}$ and the recombination to the $M_{z}^{\prime}$ state. Thus if the laser is resonant with a single $M_{z}$ to $X, M_{z}$ transition, the $M_{z}$ state is depleted, which results in a decrease of the PL coming both from the $X, M_{z}$ and the $-X, M_{z}$ transitions.

In Fig. 8 we show the result of our simulations for a dot at thermal equilibrium $\left(k_{B} T=4 \mathrm{~K}\right)$ at $t=0$, which is pumped with a laser pulse resonant with the $X=-1, M_{z}=$ $-\frac{5}{2}$ transition, which is the high-energy one, since the hole is parallel to Mn spin. The laser pulse has a duration of $300 \mathrm{~ns}$, so that the spectral broadening is negligible. In the upper panel we plot the PL coming from the counterpolarized transition, $X=+1, M_{z}=-\frac{5}{2}$, which has lower energy and can be detected without interference with the laser, for two different pumping power intensities. It is apparent that after a rise of the PL in a time scale of tens of nanoseconds, corresponding the spin relaxation of the exciton spin from $X=-1$ to $X=+1$, probably mediated by exciton-Mn exchange, the PL signal is depleted. The origin of the depletion is seen in Fig. 9. The occupation of the $M_{z}=-\frac{5}{2}$ spin state in the ground reduced down to zero, in benefit of the other Mn spin states.

Accordingly, the average magnetization becomes finite. Thus net angular momentum is transferred from the laser to the Mn spin. The transfer takes place through Mn spin relaxation enabled in the presence of the exciton. As discussed above, the most efficient mechanism combines hole-spin relaxation due to phonons combined with dark-bright mixing, which involves a Mn spin flip.

Interestingly, the fact that in the steady state several Mn spin states are occupied, including the higher energy ones, is compatible with a picture in which the Mn spin is precessing. Thus a steady supply of spin-polarized excitons in the dot would result in the precession of the Mn spin, a scenario similar to that of current drive spin-torque oscillators. ${ }^{49}$ Further work necessary to confirm this scenario is outside the scope of this paper.

The efficiency of the process increases with the laser power, as shown in Fig. 10. We define the spin orientation time $\tau_{\text {pump }}$ as the time at which the PL of the counterpolarized transition is half the maximum. We can see that, as expected, $\tau_{\text {pump }}$ is a decreasing function of the laser power. A pumping time $\tau_{\text {pump }} \simeq 90 \mathrm{~ns}$ is obtained with a generation rate of about $1 \mathrm{~ns}^{-1}$. The amplitude of the valence-band mixing, controlled by the anisotropy of the confinement potential or the in-plane strain distribution, is the main quantum dot parameter controlling the efficiency of the optical pumping. As presented

TABLE I. Parameters used in the simulation of the resonant PL observed in the time resolved optical pumping experiments.

\begin{tabular}{lcc}
\hline \hline Quantity & Symbol & Value \\
\hline Hole-Mn exchange & $j_{h}$ & $0.31 \mathrm{meV}$ \\
Electron-Mn exchange & $j_{e}$ & $-0.09 \mathrm{meV}$ \\
Electron hole & $j_{e h}$ & $-0.73 \mathrm{meV}$ \\
Uniaxial anisotropy & $D$ & $10 \mu \mathrm{eV}$ \\
In-plane anisotropy & $E$ & 0 \\
Quantum dot width & $L_{y}$ & $6 \mathrm{~nm}$ \\
Quantum dot width & $L_{x}$ & $5 \mathrm{~nm}$ \\
Quantum dot height & $L_{z}$ & $3 \mathrm{~nm}$ \\
\hline \hline
\end{tabular}

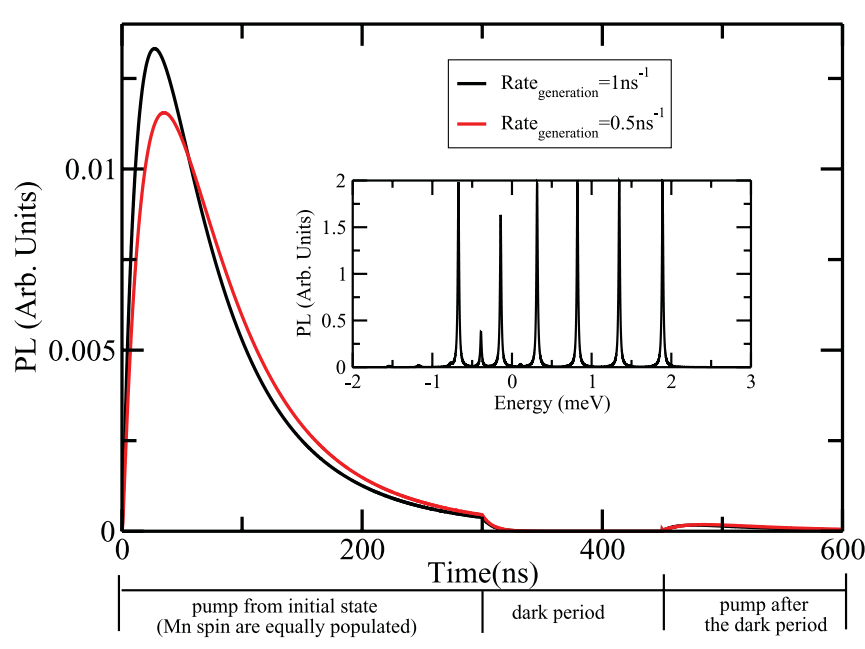

FIG. 8. (Color online) Simulation of PL intensity from state $(X=$ $+1, M_{z}=-\frac{5}{2}$ ) under the influence of a driving laser pumping the system resonantly from optical ground state $M_{z}=-\frac{5}{2}$ to the excited state $\left(X=-1, M_{z}=-\frac{5}{2}\right)$ for two laser intensities. The inset is the PL spectrum assuming all the states are equally populated. In the calculation, the quantum dot anisotropy $\left(L_{x}=5 \mathrm{~nm}\right.$ and $\left.L_{y}=6 \mathrm{~nm}\right)$ controls the LH-HH mixing. The other parameters are discussed in the text. The other parameters are shown in Table I.

in Fig. 11, decreasing the quantum dot anisotropy, i.e., decreasing the LH-HH mixing parameter $J_{h \perp}$, produces a rapid increase of $\tau_{\text {pump }}$ (inset of Fig. 11). This is a direct consequence of the reduction of the phonon induced hole spin flip.

\section{SUMMARY AND CONCLUSIONS}

We have studied the spin dynamics of a single $\mathrm{Mn}$ atom in a CdTe quantum dot excited by a laser that drives the transition between the six optical ground states, associated to the $2 S+1$ states of the Mn spin $S=\frac{5}{2}$, and the 24 single exciton states, corresponding to $X= \pm 1, \pm 2$ states interacting with the $\mathrm{Mn}$ spin. The main goal is to have a microscopic theory for the $\mathrm{Mn}$ spin-relaxation mechanisms that makes it possible to produce
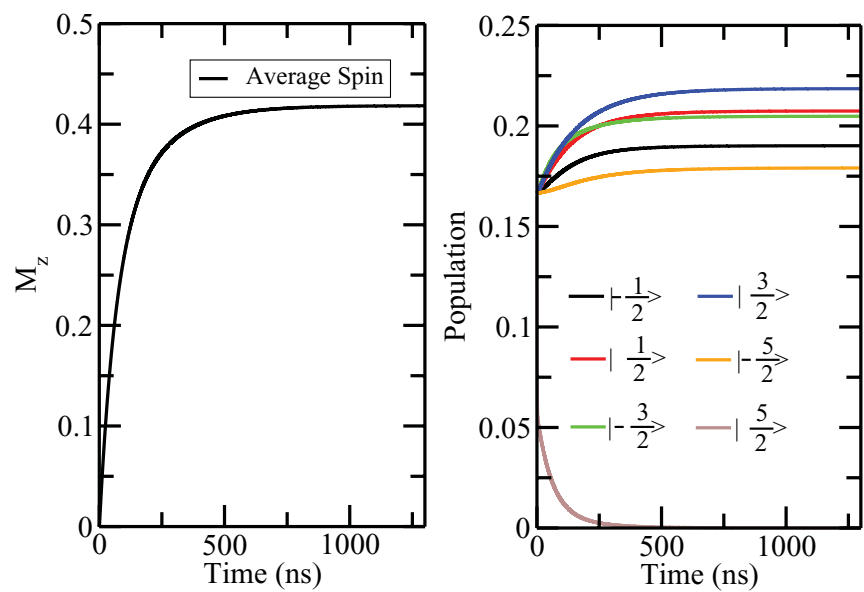

FIG. 9. (Color online) Left panel: average magnetization; right panel: occupation of the different spin states under optical pumping of the state $\left(X=-1, M_{z}=-\frac{5}{2}\right)$. Parameters are the same as for the calculation presented in Fig. 8 . 


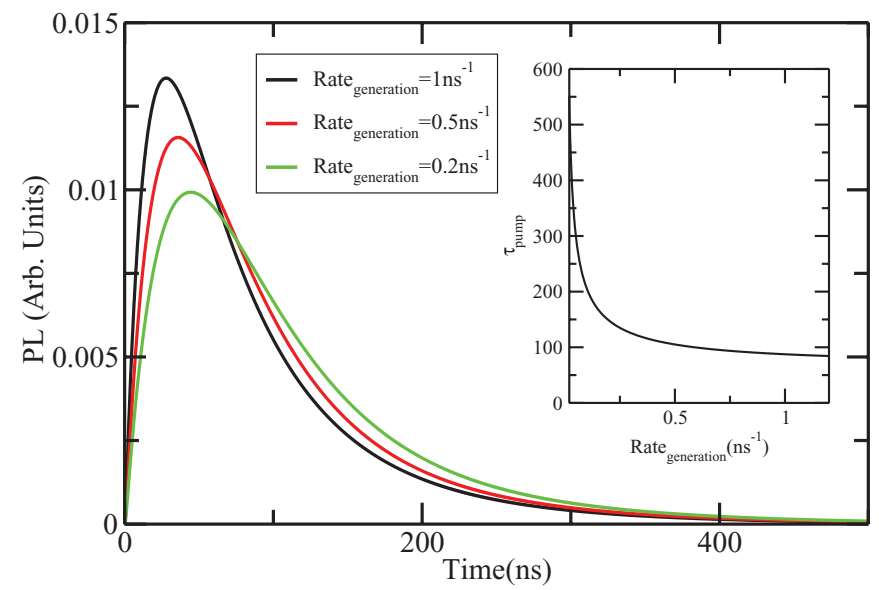

FIG. 10. (Color online) Evolution of Mn spin orientation efficiency as a function of the laser power. The pumping is detected in the PL intensity from state $\left(X=+1, M_{z}=-\frac{5}{2}\right)$ under the influence of a driving laser pumping the system from optical ground state $M_{z}=-\frac{5}{2}$ to the excited state $\left(X=-1, M_{z}=-\frac{5}{2}\right)$. The inset presents the laser power dependence of $\tau_{\text {pump }}$, from where we can see that the efficiency of the pumping gets higher with the increasing of the laser power.

laser induced Mn spin orientation in a time scale of less than $100 \mathrm{~ns}^{5-7}$ For that matter, we need to describe how the Mn and the quantum dot exciton affect each other.

In Sec. II we describe the different terms in the Mn spin Hamiltonian, including exchange with the zero-dimensional exciton. The symmetry of the exchange interaction depends on the spin properties of the carriers, which in the case of holes are strongly affected by the interplay of confinement, strain, and spin-orbit coupling. In Sec. II we also use a model for holes ${ }^{18,23,30}$ in quantum dots, which permits us to obtain

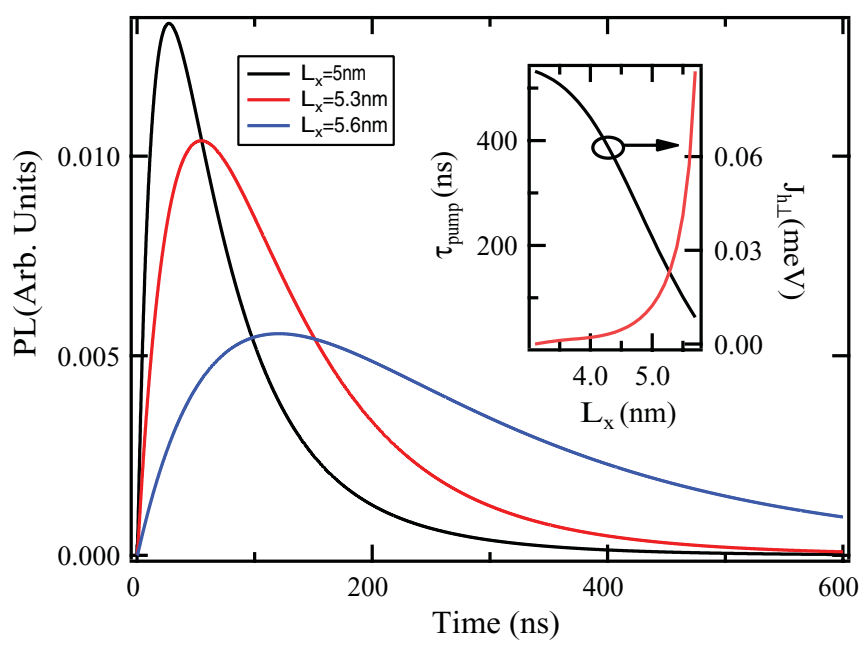

FIG. 11. (Color online) Evolution of Mn spin orientation efficiency as a function of the valence-band mixing controlled by the anisotropy of the confinement potentiel $\left(L_{y}=13 \mathrm{~nm}\right.$, variable $\left.L_{x}\right)$. The pumping is detected in the PLintensity from state $\left(X=+1, M_{z}=\right.$ $-\frac{5}{2}$ ) under the influence of a driving laser pumping the system from optical ground state $M_{z}=-\frac{5}{2}$ to the excited state $(X=-1$, $M_{z}=-\frac{5}{2}$ ). The inset shows the evolution of $\tau_{\text {pump }}$ with $L_{x}$ and $J_{h \perp}$. The exciton generation rate is fixed at $1 \mathrm{~ns}^{-1}$. analytical expressions for the wave functions of the holes, the hole-Mn exchange, in terms of the dimensions of the dot, and the Kohh-Luttinger Hamiltonian.

In Sec. III we study the dissipative dynamics of the Mn spin due to its coupling to phonons, both with and without excitons in the dot. The Mn spin-phonon coupling arises from the time-dependent stochastic fluctuations of the crystal field and thereby of the single-ion magnetic anisotropy, induced by the phonon field. Whereas the Mn spin relaxation is accelerated by two or three orders of magnitude in the presence of the exciton, the efficiency of this mechanism is too low to account for the optical orientation of the Mn spin reported experimentally. ${ }^{5-7}$ The small Mn spin-phonon coupling comes from the small magnetic anisotropy of $\mathrm{Mn}$ as a substituional impurity in CdTe.

In Sec. IV we describe the interaction between the hole spin and the phonons in nonmagnetic dots. Using the simple analytical model for the holes presented in Sec. II we obtain analytical formulas for the hole spin relaxation. We find that hole spin lifetime can be in the range of $30 \mathrm{~ns}$ for a hole spin splitting as large as that provided by the hole-Mn coupling. Thus we expect that bright excitons will relax into dark excitons via hole-spin relaxation. This provides a microscopic mechanism to the scenario for Mn spin relaxation proposed by Cywinski: ${ }^{29}$ bright excitons relax into dark excitons, via carrier spin relaxation, and the joint process of Mn-carrier spin exchange couples the dark excitons to the bright excitons, resulting in PL from dark states, which implies Mn spin relaxation in a time scale of a few nanoseconds. This scenario is confirmed by calculations presented in Sec. V. Finally, in Sec. VI we present the master equation that governs the dynamics of the 30 states of the dot, we solve it numerically and we model the optical Mn spin orientation reported experimentally.

Our main conclusions are

(1) Mn spin-phonon spin relaxation is presumably too weak to account for Mn spin dynamics in the presence of the exciton.

(2) The Mn spin orientation is possible in a time scale of $100 \mathrm{~ns}$ via a combination of phonon induced hole spin relaxation and the subsequent recombination of the dark exciton enabled by spin-flip exchange of the Mn and the carrier.

(3) The critical property that governs the hole-Mn exchange and the hole spin relaxation is the mixing between light and heavy holes, which depends both on the shape of the dot and on strain.

(4) Our microscopic model permits us to account for the optically induced Mn spin orientation.

Future work should address how the coupling of the electronic Mn spin to the nuclear spin modifies our results. This probably plays a role for the $\mathrm{Mn}$ in the dot without excitons. In addition, future work should study the role played by $\mathrm{Mn}$ spin coherence, and the interplay between optical and spin coherence.

\section{ACKNOWLEDGMENTS}

We thank F. Delgado, C. Le Gall, R. Kolodka, and H. Mariette for fruitful discussions. This work has been financially supported by MEC-Spain (Grants No. MAT07-67845, No. FIS2010-21883-C02-01, and No. 
CONSOLIDER CSD2007-00010), Generalitat Valenciana (Grant No. ACOMP/2010/070), Foundation NanoScience (RTRA Genoble), and French ANR Contract No. QuAMOS.

\section{APPENDIX A: KOHN LUTTINGER HAMILTONIAN}

The Kohn-Luttinger Hamiltonian for the four topmost valence bands of a zinc-blende compound are given by

$$
\mathcal{H}\left(k_{x}, k_{y}, k_{z}\right)=\left(\begin{array}{lccc}
P+Q-\frac{3}{2} \kappa \nu_{B} B & S & R & 0 \\
S^{\dagger} & P-Q-\frac{1}{2} \kappa \mu_{B} B & 0 & R \\
R^{\dagger} & 0 & P-Q+\frac{1}{2} \kappa \mu_{B} B & -S \\
0 & R^{\dagger} & -S^{\dagger} & P+Q+\frac{3}{2} \kappa v_{B} B
\end{array}\right)
$$

where $^{35}$

$$
\begin{gathered}
P=\hbar^{2} \gamma_{1} \frac{k_{z}^{2}+k_{\perp}^{2}}{2 m_{0}} Q=\hbar^{2} \gamma_{1} \frac{-2 k_{z}^{2}+k_{\perp}^{2}}{2 m_{0}}, \\
S=2 \sqrt{3} \gamma_{2} \frac{\hbar^{2} k_{z} k_{\perp}}{2 m_{0}},
\end{gathered}
$$

and

$$
R=-\frac{\sqrt{3} \hbar^{2}}{2 m_{0}}\left(-\bar{\gamma} k_{-}^{2}+\mu k_{+}^{2}\right),
$$

where $\gamma_{1,2,3}$ are dimensionless material dependent parameters, $\bar{\gamma}=\frac{1}{2}\left(\gamma_{2}+\gamma_{3}\right), \mu=\frac{1}{2}\left(\gamma_{2}-\gamma_{3}\right), m_{0}$ is the free-electron mass, $k_{\perp}^{2}=k_{x}^{2}+k_{y}^{2}$, and $k_{ \pm}=k_{x} \pm i k_{y}$.

Using the notation of Eq. (3), the matrices $V_{i j}^{\mathrm{KL}}(\vec{k})$ read

$$
\begin{aligned}
V_{x x}=V_{y y} & =\frac{4}{15} P-\frac{5}{4} Q+\frac{1}{2 \sqrt{3}}\left(R+R^{*}\right), \\
V_{x y} & =V_{y x}=\frac{-i}{2 \sqrt{3}}\left(R-R^{*}\right), \\
V_{x z} & =V_{z x}=\frac{-1}{2 \sqrt{3}}\left(S+S^{*}\right), \\
V_{y z} & =V_{z y}=\frac{-i}{2 \sqrt{3}}\left(S-S^{*}\right), \\
V_{z z} & =\frac{4}{15} P-\frac{1}{4} Q .
\end{aligned}
$$

For the dot states the relevant parameters are

$$
\begin{gathered}
\bar{P}=\frac{\hbar^{2}}{2 m_{0}} \gamma_{1} \pi^{2}\left(\frac{1}{L_{z}^{2}}+\frac{1}{L_{x}^{2}}+\frac{1}{L_{y}^{2}}\right), \\
\bar{Q}=\frac{\hbar^{2} \gamma_{1}}{2 m_{0}} \pi^{2}\left(\frac{-2}{L_{z}^{2}}+\frac{1}{L_{x}^{2}}+\frac{1}{L_{y}^{2}}\right), \\
\bar{R}=-\frac{\hbar^{2} \pi^{2}}{2 m_{0}} \sqrt{3} \gamma_{2}\left(\frac{1}{L_{x}^{2}}-\frac{1}{L_{y}^{2}}\right) .
\end{gathered}
$$

\section{APPENDIX B: GENERAL FORMULA FOR PHONON INDUCED SPIN-FLIP RATE}

In this Appendix we derive a general formula for the scattering rate between two electronic states $n$ and $n^{\prime}$ induced by a phonon emission. The Hamiltonian of the system can be split in three parts: the electronic states $n$, the phonon states, and their mutual coupling. The phonon states are labeled according to their polarization and momentum, $\lambda, \vec{q}$. We consider the following coupling:

$$
\mathcal{V}=\sum_{m, m^{\prime}, \vec{q}, \lambda} \mathcal{V}_{\vec{q}, \lambda}^{m, m^{\prime}}|m\rangle\left\langle m^{\prime}\right|\left(b_{\lambda q}^{\dagger}+b_{\lambda,-q}\right),
$$

where $m$ and $m^{\prime}$ are electronic states. We refer to the free phonon states as the reservoir states. Within the Born-Markov approximation, the scattering rate between states $n$ and $n^{\prime}$ is

$$
\begin{array}{r}
\Gamma_{n \rightarrow n^{\prime}}=\frac{2 \pi}{\hbar} \sum_{r} P_{r} \sum_{r^{\prime}}\left|\left\langle n r|V| n^{\prime} r^{\prime}\right\rangle\right|^{2} \\
\times \delta\left(E_{n}-E_{n^{\prime}}+e_{r}-e_{r^{\prime}}\right),
\end{array}
$$

where $P_{r}$ is the occupation of the $r$ reservoir state with energy $e_{r}$. This equation can be interpreted as a statistical average over reservoir initial states $r$ of the Fermi golden rule decay rate of state $N, r$.

The sums over $r$ and $r^{\prime}$ are performed using the following trick. For a given $r$, the initial reservoir state, $r^{\prime}$ must have an additional phonon, since we consider the phonon emission case. Thus we write

$$
\left|r^{\prime}\right\rangle=\frac{1}{\sqrt{n_{\lambda^{\prime}, q^{\prime}}+1}} b_{\lambda^{\prime}, q^{\prime}}^{\dagger}|r\rangle
$$

so that

$$
\left\langle r\left|b_{q, \lambda}^{\dagger}+b_{-q, \lambda}\right| r^{\prime}\right\rangle=\delta_{-q, q^{\prime}} \delta_{\lambda, \lambda^{\prime}} \sqrt{n_{\lambda^{\prime}, q^{\prime}}^{r}+1}
$$

The matrix element

$$
\left\langle n r|V| n^{\prime} r^{\prime}\right\rangle=\mathcal{V}_{\vec{q}, \lambda}^{n, n^{\prime}} \sqrt{n_{\lambda^{\prime}, q^{\prime}}^{r}+1}
$$

We see how from all the terms in the sum that defines the coupling, only one survives and fixes the index $r^{\prime}$. Thus the only sums left are over the initial reservoir states and the $\lambda, q$ index that define the final state. Now we use the definition of the Bose function,

$$
\sum_{r} P_{r}\left(n_{\lambda^{\prime}, q^{\prime}}^{r}+1\right)=n_{B}\left[\omega_{\lambda^{\prime}}\left(q^{\prime}\right)\right]+1,
$$


and we arrive at the following expression for the rate:

$$
\begin{gathered}
\Gamma_{n \rightarrow n^{\prime}}=\frac{2 \pi}{\hbar} \sum_{\lambda, q}\left|\mathcal{V}_{\vec{q}, \lambda}^{n, n^{\prime}}\right|^{2}\left\{n_{B}\left[\omega_{\lambda}(q)\right]+1\right\} \\
\times \delta\left[E_{n}-E_{n^{\prime}}-\omega_{\lambda}(q)\right] .
\end{gathered}
$$

Notice that it is possible to write the rate as a sum over different contributions arising from different polarizations, $\Gamma=\sum_{\lambda} \Gamma_{\lambda}$.
In the particular case that we can neglect the dependence of the matrix element $\mathcal{V}_{\vec{q}, \lambda}\left(n, n^{\prime}\right) \simeq\left|\mathcal{V}_{\left(n, n^{\prime}\right)}\right|^{2}$ on $\vec{q}$ and $\lambda$, we arrive at the following expression:

$$
\Gamma_{n \rightarrow n^{\prime}}=\frac{2 \pi}{\hbar}\left[n_{B}(\Delta)+1\right] \rho_{\lambda}(\Delta)\left|\mathcal{V}_{n, n^{\prime}}\right|^{2},
$$

where $\rho(\Delta) \equiv \sum_{\lambda, q} \delta\left[\Delta-\omega_{\lambda}(q)\right]$ is the density of states of the phonons evaluated at the transition energy $\Delta$.
${ }^{1}$ P. M. Koenraad and M. E. Flatte, Nature (London) 10, 91 (2011). ${ }^{2}$ C. Hirjibehedin, C.-Y. Lin, A. Otte, M. Ternes, C. P. Lutz, B. A. Jones, and A. J. Heinrich, Science 317, 1199 (2007).

${ }^{3}$ S. Loth, K. von Bergmann, M. Ternes, A. F. Otte, C. P. Lutz, and A. J. Heinrich, Nat. Phys. 6, 340 (2010).

${ }^{4}$ F. Jelezko, T. Gaebel, I. Popa, A. Gruber, and J. Wrachtrup, Phys. Rev. Lett. 92, 076401 (2004).

${ }^{5}$ C. Le Gall, L. Besombes, H. Boukari, R. Kolodka, J. Cibert, and H. Mariette, Phys. Rev. Lett. 102, 127402 (2009).

${ }^{6}$ M. Goryca, T. Kazimierczuk, M. Nawrocki, A. Golnik, J. A. Gaj, P. Kossacki, P. Wojnar, and G. Karczewski, Phys. Rev. Lett. 103, 087401 (2009).

${ }^{7}$ C. Le Gall, R. S. Kolodka, C. L. Cao, H. Boukari, H. Mariette, J. Fernández-Rossier, and L. Besombes, Phys. Rev. B 81, 245315 (2010).

${ }^{8}$ L. Besombes, Y. Léger, L. Maingault, D. Ferrand, H. Mariette, and J. Cibert, Phys. Rev. Lett. 93, 207403 (2004).

${ }^{9}$ L. Besombes, Y. Leger, L. Maingault, D. Ferrand, H. Mariette, and J. Cibert, Phys. Rev. B 71, 161307 (2005).

${ }^{10}$ Y. Léger, L. Besombes, L. Maingault, D. Ferrand, and H. Mariette, Phys. Rev. Lett. 95, 047403 (2005).

${ }^{11}$ Y. Léger, L. Besombes, L. Maingault, D. Ferrand, and H. Mariette, Phys. Rev. B 72, 241309 (2005).

${ }^{12}$ Y. Léger L. Besombes, J. Fernández-Rossier, L. Maingault, and H. Mariette, Phys. Rev. Lett. 97, 107401 (2006).

${ }^{13}$ Y. Léger, L. Besombes, L. Maingault, and H. Mariette, Phys. Rev. B 76, 045331 (2007).

${ }^{14}$ L. Besombes, Y. Leger, J. Bernos, H. Boukari, H. Mariette, J. P. Poizat, T. Clement, J. Fernández-Rossier, and R. Aguado, Phys. Rev. B 78, 125324 (2008).

${ }^{15}$ M. Goryca, P. Plochocka, T. Kazimierczuk, P. Wojnar, G. Karczewski, J. A. Gaj, M. Potemski, and P. Kossacki, Phys. Rev. B 82, 165323 (2010).

${ }^{16}$ A. Kudelski, A. Lemaitre, A. Miard, P. Voisin, T. C. M. Graham, R. J. Warburton, and O. Krebs, Phys. Rev. Lett. 99, 247209 (2007).

${ }^{17}$ O. Krebs, E. Benjamin, and A. Lemaître, Phys. Rev. B 80, 165315 (2009).

${ }^{18}$ J. Fernández-Rossier, Phys. Rev. B 73, 045301 (2006).

${ }^{19}$ F. Qu and P. Hawrylak, Phys. Rev. Lett. 95, 217206 (2005).

${ }^{20}$ A. O. Govorov, Phys. Rev. B 70, 035321 (2004).

${ }^{21}$ A. K. Bhattacharjee and J. Pérez-Conde, Phys. Rev. B 68, 045303 (2003).

${ }^{22}$ A. K. Bhattacharjee, Phys. Rev. B 76, 075305 (2007).

${ }^{23}$ D. E. Reiter, T. M. Kuhn, and V. M. Axt, Phys. Rev. Lett. 102, 177403 (2009).

${ }^{24}$ D. E. Reiter, T. M. Kuhn, and V. M. Axt, Phys. Rev. B 83, 155322 (2011).
${ }^{25}$ J. Fernández-Rossier and L. Brey, Phys. Rev. Lett. 93, 117201 (2004).

${ }^{26}$ J. van Bree, P. M. Koenraad, and J. Fernández-Rossier, Phys. Rev. B 78, 165414 (2008).

${ }^{27}$ A. O. Govorov and A. V. Kalameitsev, Phys. Rev. B 71, 035338 (2005).

${ }^{28}$ C. L. Cao, L. Besombes, and J. Fernandez-Rossier, J. Phys.: Conf. Ser. 210, 012046 (2010).

${ }^{29}$ L. Cywinski, Phys. Rev. B 82, 075321 (2010).

${ }^{30}$ F. V. Kyrychenko and J. Kossut, Phys. Rev. B 70, 205317 (2004).

${ }^{31}$ L. M. Woods, T. L. Reinecke, and R. Kotlyar, Phys. Rev. B 69, 125330 (2004).

${ }^{32}$ J. K. Furdyna, J. Appl. Phys. 64, R29 (1988).

${ }^{33}$ M. Qazzaz, G. Yang, S. H. Xin, L. Montes, H. Luo, and J. K. Furdyna, Solid State Commun. 96, 405 (1995).

${ }^{34}$ J. M. Luttinger and W. Kohn, Phys. Rev. 97, 869 (1955).

${ }^{35}$ D. A. Broido and L. J. Sham, Phys. Rev. B 31, 888 (1985).

${ }^{36}$ Lok C. Lew Yan Voon and Morten Willatzen, The $k \cdot p$ Method: Electronic Properties of Semiconductors (Springer, New York, 2009).

${ }^{37}$ Peter Yu and Manuel Cardona, Fundamentals of Semiconductors (Springer, New York, 1996).

${ }^{38}$ A. K. Bhattarjee and C. Benoit a la Guillaume, Solid State Commun. 113, 17 (2000).

${ }^{39}$ J. Fernández-Rossier and R. Aguado, Phys. Rev. Lett. 98, 106805 (2007).

${ }^{40}$ E. M. Chudnovsky, D. A. Garanin, and R. Schilling, Phys. Rev. B 72, 094426 (2005)

${ }^{41}$ J. C. Merle, R. Sooryakumar, and M. Cardona, Phys Rev. B 30, 3261 (1984).

${ }^{42}$ J. G. Collins, G. K. White, J. A. Birch, and T. F. Smith, J. Phys. C 13, 1649 (1980).

${ }^{43}$ T. Strutz, A. M. Witowski, and P. Wyder, Phys. Rev. Lett. 68, 3912 (1992).

${ }^{44}$ E. Tsitsishvili, R. v. Baltz, and H. Kalt, Phys. Rev. B 72, 155333 (2005).

${ }^{45}$ K. Roszak, V. M. Axt, T. Kuhn, and P. Machnikowski, Phys. Rev. B 76, 195324 (2007).

${ }^{46}$ W. H. Kleiner and L. M. Roth, Phys. Rev. B 2, 334 (1959).

${ }^{47}$ U. Bockelmann, Phys. Rev. B 50, 17271 (1994).

${ }^{48}$ Al. V. Khaetskii and Y. V. Nazarov, Phys. Rev. B 64, 125316 (2001).

${ }^{49}$ S. I. Kiselev, J. C. Sankey, I. N. Krivorotov, N. C. Emley, R. J. Schoelkopf, R. A. Buhrman, and D. C. Ralph, Nature (London) 425, 380 (2003). 